REVISTA

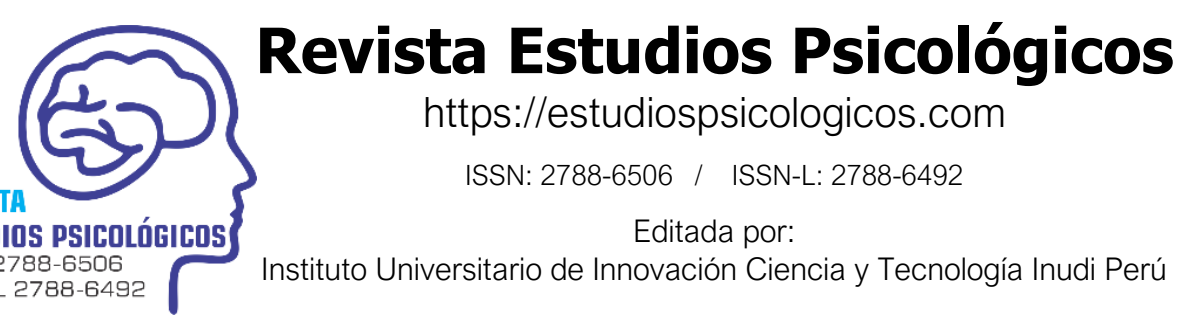

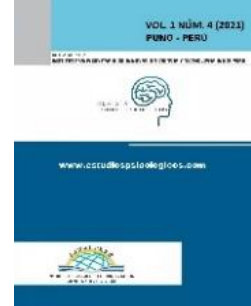

ARTÍCULO ORIGINAL

\title{
Desarrollo de competencias científicas en estudiantes de educación básica secundaria y media como propósito de la gestión pedagógica
}

\author{
Development of scientific competences in students of basic secondary and secondary education as a \\ purpose of pedagogical management
}

Desenvolvimento de competências científicas em alunos do ensino médio e fundamental como objetivo da gestão pedagógica

Francisco Blanchar ${ }^{1}$

Universidad Tecnológica de Pereira UTP, Pereira - Risaralda, Colombia

(iD) https://orcid.org/0000-0002-0176-8551

D0I: https://doi.org/10.35622/j.rep.2022.02.003

Enviado: 25/12/2021/ Aceptado: 28/03/2022

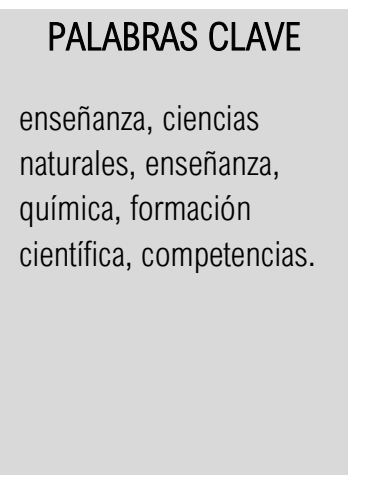

KEYWORDS
teaching, natural
sciences, teaching,
chemistry, scientific
training, skills.

RESUMEN. El presente artículo se deriva de la investigación realizada en la institución educativa oficial Centro de Integración Popular IPC del distrito turístico de Riohacha, cuyo objetivo fue analizar el nivel de incidencia de las prácticas pedagógicas de química en el desarrollo de competencias científicas de estudiantes de educación básica secundaria. El diseño de la investigación fue no experimental, de tipo transversal. Para la evaluación del desarrollo de las competencias científicas se utilizó una rejilla de evaluación de las competencias científicas a partir de cuestionarios de preguntas estandarizadas de lápiz y papel diseñados en función de las pruebas censales Saber Icfes. Para el análisis de la información se utilizó estadística descriptiva, los resultados permitieron rechazar la hipótesis de trabajo, en el sentido de afirmar que las prácticas pedagógicas del área de química de la institución educativa promueven de manera limitada el desarrollo pertinente de competencias científicas.

ABSTRACT. This article derives from the research carried out in the official educational institution Centro of Integration Popular IPC of the tourist district of Riohacha, whose objective was to analyze the level of incidence of the pedagogical practices of chemistry in the development of scientific competences of students of basic education secondary. The research design was non-experimental, cross-sectional. For the evaluation of the development of scientific competences, a grid for the evaluation of scientific competences was used from questionnaires of standardized pencil and paper questions designed based on the Saber Icfes census tests. For the analysis of the information, descriptive statistics were used, the results allowed rejecting the working hypothesis, in the sense of affirming that the pedagogical practices of the chemistry area of the educational institution promote in a limited way the pertinent development of scientific competences.

\footnotetext{
${ }^{1}$ Correspondencia: Francisco.blanchar@utp.edu.co
} 
PALAVRAS-CHAVE

ensino, ciências naturais, ensino, química, formação científica, competências.
RESUMO. Este artigo deriva da pesquisa realizada na instituição educacional oficial Centro de Integração Popular IPC do bairro turístico de Riohacha, cujo objetivo foi analisar o nível de incidência das práticas pedagógicas de química no desenvolvimento de competências científicas de alunos do ensino fundamental. educação secundária. 0 desenho da pesquisa foi não experimental, transversal. Para a avaliação do desenvolvimento das competências científicas, foi utilizada uma grade de avaliação das competências cientificas a partir de questionários de questões padronizadas de lápis e papel elaboradas com base nas provas censitárias Saber Icfes. Para a análise das informações, utilizou-se estatística descritiva, os resultados permitiram rejeitar a hipótese de trabalho, no sentido de afirmar que as práticas pedagógicas da área de química da instituição de ensino promovem de forma limitada o desenvolvimento pertinente de competências científicas.

\section{INTRODUCCIÓN}

Como fenómeno multidimensional y complejo la educación ha establecido el propósito presente de la formación y desarrollo intelectual de los individuos mediante el análisis, enseñanza, aprendizaje, así como la construcción de diversos conocimientos derivados de las distintas áreas del saber. Aspectos como la construcción de ciudadanía, la formación de individuos conscientes de su responsabilidad y compromiso con el desarrollo del grupo social al que pertenecen hacen parte de las metas formativas de todas las disciplinas vinculadas al sistema educativo. En este sentido, existe un consenso histórico relacionado con la importancia de la educación como estandarte fundamental del desarrollo social. Tal como lo plantea la Comisión Internacional sobre la Educación para el Siglo XXI en su informe a la UNESCO, al señalar que, frente a los numerosos desafíos del porvenir, la educación constituye un instrumento indispensable para que la humanidad pueda progresar hacia los ideales de paz, libertad y justicia social (Delors et al., 1996).

El papel trascendental de la educación para la sociedad, puesta en práctica en los sus sistemas educativos a nivel mundial, específicamente en materia científica. Al respecto la (Delors et al., 1996) ha establecido necesidad de formar integralmente a los individuos de las sociedades en función del desarrollo de tres conjuntos de habilidades del sujeto: el saber, el saber hacer y el ser, denominadas competencias.

El saber relacionado con las competencias conceptuales (dominio de contenidos teóricos y formal en la especialidad), el saber hacer relacionado con las competencias procedimentales (manejo de las herramientas didácticas y aplicación de metodología de enseñanza) y el ser o competencias actitudinales.

En este marco Tobón et al. (2010) manifiestan que estas directrices establecidas a nivel mundial deben ser adaptarlas a sus contextos y particularidades, a partir de propuestas de formación alineadas con los retos del contexto y las políticas educativas vigentes.

El Ministerio de Educación Nacional de Colombia (MEN, 2014) ha apropiado la tendencia a la formación por competencias básicas, ciudadanas y laborales, enmarcada en el cumplimiento de metas educativas basadas en la cobertura de todos los estudiantes.

La estrategia definida en el país como punta de lanza para alcanzar los objetivos propuestos se denomina ciclo de calidad, basada en la correlación de tres aspectos:1) La formulación de un conjunto de competencias cuya 
complejidad y especialización crece en la medida en que se alcanzan mayores niveles de educación, denominadas Estándares básicos de competencia. 2) El establecimiento de procesos de evaluación de los estudiantes, directivos docentes, docentes e instituciones educativas, como actividad de fortalecimiento que permita establecer el desempeño en el cual se hacen evidentes los conocimientos, aptitudes, capacidades, habilidades y actitudes de los estudiantes producto de las actividades formativas. 3) El mejoramiento continuo, sustentado en que la educación solo puede ser mejorada y con mayor calidad, si se puede establecer en donde está cada proceso y como está mejorando en relación a las metas propuestas y los resultados esperados (MEN, 2008).

A nivel de las ciencias naturales, todos los aspectos relevantes de la vida cotidiana del ser humano se relacionan con el uso del conocimiento científico, en este sentido parece difícil que este logre comprender el mundo y desenvolverse en él sin una formación científica básica en los diferentes niveles de educación, en torno al desarrollo de competencias científicas. Por lo tanto, su formación requiere del desarrollo de competencias pertinentes vinculadas con este saber y relacionadas estrechamente son su contexto, en ámbitos tan cruciales como el transporte, la democracia, las comunicaciones, la toma de decisiones, la alimentación, la medicina, el entretenimiento, las artes e, inclusive, la educación, están signados por los avances científicos y tecnológicos (MEN, 2006).

Al respecto, las competencias científicas representan capacidades para la identificación, interpretación, estudio, análisis y comprensión de los fenómenos naturales se definen a través de los denominados Estándares Básicos de Competencias en Ciencias Naturales, a fin de llevar vidas responsables cuyas actuaciones estén a favor de sí mismos y de la sociedad en su conjunto.

Es así como desde el ámbito escolar, la enseñanza de la química se ha establecido como una de las principales ciencias naturales que permitiría contribuir a la consolidación de competencias científicas basadas en la comprensión, explicación y entendimiento de los principales fenómenos naturales y sociales que constituyen el entorno cotidiano de los seres humanos, y consolidar la capacidad reflexiva y crítica de los sujetos para tomar decisiones apropiadas en torno al desarrollo económico, político, social, ambiental y ecológico de la sociedad (Tobón et al., 2010).

Investigadores como Davini (2015) y Litwin (2008) destacan el papel trascendental de la enseñanza y los enfoques educativos en las prácticas pedagógicas institucionales para el logro de los objetivos educativos propuestos.

\section{Planteamiento del problema}

Se han promovido diversas iniciativas orientadas a la enseñanza de las ciencias naturales a nivel mundial, por ejemplo, la promoción desde los años 70 de propuestas enmarcadas en la indagación guiada, ampliamente promovida en los Estados Unidos mediante la financiación de la NSF (National Science Foundation), extendida hasta los años 80 del siglo XX. En Francia se desarrolló el proyecto La main à la pâte1 (Manos a la obra), con éxito en la renovación de la enseñanza de las ciencias en la escuela primaria (Hernandes et al., 2004). 
A nivel nacional, en Colombia se han presentado un conjunto de programas, proyectos e iniciativas de carácter públicas y privadas, por ejemplo el proyecto conjunto entre las instituciones Maloka, la Universidad de los Andes, el Liceo Francés Louis Pasteur y la Alianza Educativa, que orientados bajo las iniciativas internacionales incentivaron desde el año 2000 un movimiento de reforma en la educación en ciencias naturales, mediante el programa denominado Pequeños Científicos, con el objetivo principal estimular y contribuir a la renovación de la enseñanza-aprendizaje de las ciencias naturales en instituciones educativas en Colombia.

Sumado a lo anterior se encuentra el trabajo del Departamento Administrativo de Ciencia, Tecnología e Innovación COLCIENCIAS (hoy MinCiencias) a través del Programa Ondas, que incentivó la formación y promoción de la capacidad de investigación en ciencias en niños, niñas y jóvenes en edad escolar mediante el financiamiento de proyectos de investigación bajo la tutoría de los docentes.

Por otra parte, existen una serie de orientaciones y directrices pedagógicas y normativas como apoyo al fortalecimiento de la enseñanza de las ciencias: las secuencias didácticas en ciencias naturales educación básica primaria para el sector educativo rural PER II (Arbeláez Sánchez et al., 2013) y las secuencias didácticas desde el grado transición hasta el grado $11^{\circ}$ de educación media vocacional (MEN, 2017).

Normativamente, derivado de la Ley 115 o Ley General de Educación (Congreso de la República de Colombia, 1994), se encuentra el Decreto 1743 de 1994 del MEN (1994), que establece el Proyecto de Educación Ambiental para todos los niveles de educación formal, sumado a los Planes Decenales de Educación. Además de los Lineamientos Curriculares de Ciencias Naturales y Educación Ambiental (MEN, 1998), los Estándares Básicos de Competencia de ciencias naturales (MEN, 2006), Ios Derechos Básicos de Aprendizaje DBA de ciencias naturales (MEN, 2016), las Matrices de Referencia de ciencias naturales (MEN, 2016b).

Sin embargo, a pesar de todo el conjunto de políticas públicas, programas, proyectos y directrices orientadoras implementadas en materia de educación científica la realidad educativa difiere de las metas y objetivos propuestos, teniendo en cuenta que pues a pesar de los esfuerzos de todos los entes involucrados en el ámbito educativo relacionados anteriormente, se evidencia que producto de las practicas pedagógicas de enseñanza de las ciencias naturales tradicionales y enmarcadas en enfoques de promoción de la memoria, la repetición conceptual, declarativa, que generan aprendizajes deficientes y limitados en materia de las competencias científicas como el uso comprensivo del conocimiento científico, la explicación de fenómenos y la indagación, requeridas para que los sujetos puedan desempeñarse, comprender el mundo, además de participar de manera activa en su construcción.

La evaluación de los aprendizajes, tanto en el ámbito de pruebas internacionales, como nacionales e internas en los establecimientos educativos, dan cuenta de la problemática relacionada con la criticidad de la formación científica. Evidenciada en los resultados en pruebas internacionales como PISA (Programa Internacional de Evaluación de Estudiante), durante los años 2006, 2012, 2016 y 2018, que en el área de ciencias detectó débiles desempeños que los estudiantes de los grados $7^{\circ}$ y $11^{\circ}$ cuando se enfrentan a un problema complejo de la vida real que requiere solución (Banco Mundial, 2008). 
Los resultados en el área de ciencias, reitera el Banco Mundial, han sido los más bajos, teniendo en cuenta que el país no cumple con el esperado según su nivel de ingreso per cápita, pues otros países de ingresos similares al colombiano, como Jordania e Indonesia, logran superar los promedios asociados a su Producto Interno Bruto. En las aplicaciones históricas de la prueba en el área de ciencias, la calificación media de Colombia ha quedado por debajo de todos sus comparadores regionales, levemente por debajo de Brasil, y Argentina y debajo de México, Uruguay y Chile.

Por su parte, de acuerdo a los resultados de los estudios regionales, comparativos y explicativos de (ERCE, SERCE y TERCE) promovidos por la Organización de las Naciones Unidades para la Educación UNESCO (2013), que evalúan los procesos de reconocimiento de conceptos, la aplicación de conceptos e interpretación y solución de problemas en el ámbito científico en tres dominios conceptuales (seres vivos, salud, tierra, ambiente, materia y energía) de estudiantes de grado sexto de educación básica secundaria, Ios estudiantes de Colombia han demostrado un leve mejoramiento teniendo en cuenta la disminución del porcentaje ubicado en el Nivel I (precariedad), más bajo de la prueba, el cual se redujo de 9,15\% en el SERCE a 3,4\% en el TERCE, aún se siguen percibiendo rezagos en las habilidades científicas de los estudiantes de Colombia y de américa Latina.

Estos resultados en materia de educación científica de los estudiantes colombianos indican marcadas limitaciones en el uso de las habilidades y/o competencias básicas de carácter científico, que les permita analizar y resolver correctamente situaciones problémicas complejas a la luz de las ciencias. Se vinculan como consecuencia de acciones de enseñanza tradicionales en torno a la transmisión de información insustancial y además de premiar su memorización, aspectos que requieren el cambio de percepciones y creencias de los maestros en su forma de enseñar (Instituco Colombiano para la Evaluación de la Calidad de la Educación ICFES, 2016).

Esta misma perspectiva de resultados deficientes se presenta a nivel nacional, de acuerdo a los resultados de las pruebas estandarizadas de lápiz y papel de carácter censal y muestral SABER ICFES, del área de ciencias naturales, aplicadas a los grados $5^{\circ}$ de educación básica primaria, $9^{\circ}$ de educación básica secundaria y grado $11^{\circ}$ de educación media vocacional.

A nivel nacional, los resultados de pruebas nacionales en ciencias para los grados $5^{\circ}$ de educación básica primaria y $9^{\circ}$ de educación básica secundaria (2009, 2012, 2014 y 2016), se evidencia, tal como lo muestra la información de la tabla 1, un aumento progresivo del puntaje promedio de los estudiantes de grado $5^{\circ}$ y una disminución paulatina del porcentaje estudiantil ubicada inicialmente en los niveles de menor desempeño de las pruebas (insuficiente y mínimo). Sin embargo, cabe resaltar que en todas las pruebas aplicadas en promedio el $60,1 \%$ aproximadamente se mantiene en los niveles menores de la escala de valoración. 


\section{Tabla 1}

Resultados históricos a nivel nacional de la prueba SABER de ciencias naturales grado $5^{\circ}$

\begin{tabular}{llllll}
\hline \multirow{2}{*}{ Aplicación } & $\begin{array}{l}\text { Puntaje } \\
\text { promedio }\end{array}$ & $\begin{array}{l}\text { \% de estudiantes en } \\
\text { nivel insuficiente }\end{array}$ & $\begin{array}{l}\text { \% de estudiantes desempeño } \\
\text { en nivel mínimo }\end{array}$ & $\begin{array}{l}\text { nivel satisfactorio } \\
\text { niviantes en }\end{array}$ & $\begin{array}{l}\% \text { de estudiantes } \\
\text { en nivel avanzado }\end{array}$ \\
2009 & 310,87 & $16,13 \%$ & $47,55 \%$ & $23,66 \%$ & $12,81 \%$ \\
2012 & 311,52 & $17,88 \%$ & $44,79 \%$ & $22,91 \%$ & $14,59 \%$ \\
2014 & 318,03 & $13,57 \%$ & $49,47 \%$ & $21,45 \%$ & $15,67 \%$ \\
2016 & 328,15 & $11,17 \%$ & $43,86 \%$ & $26,73 \%$ & $18,39 \%$ \\
\hline
\end{tabular}

Nota. Esta tabla muestra que a diferencia del grado $5^{\circ}$, los resultados de los estudiantes del grado $9^{\circ}$ (ver tabla 2) han presentado un comportamiento irregular, entre un aumento entre las pruebas de 2009 y 2012, y la disminución progresiva del puntaje promedio de la aplicación 2014 y 2016, similar comportamiento en la distribución histórica de la población estudiantil en los niveles de menor desempeño y de mayor desempeño. El porcentaje promedio de los estudiantes del grado $9^{\circ}$ ubicados en los niveles insuficiente y mínimo es del $64,4 \%$.

\section{Tabla 2}

Resultados históricos a nivel nacional prueba SABER de ciencias naturales grado $9^{\circ}$

\begin{tabular}{llllll}
\hline \multirow{2}{*}{ Aplicación } & $\begin{array}{l}\text { Puntaje } \\
\text { promedio }\end{array}$ & \multicolumn{4}{c}{ Niveles de desempeño } \\
& & $\begin{array}{l}\text { \% de estudiantes en } \\
\text { nivel insuficiente }\end{array}$ & $\begin{array}{l}\text { \% de estudiantes } \\
\text { en nivel mínimo }\end{array}$ & $\begin{array}{l}\text { nivel satisfactorio } \\
\text { nivel }\end{array}$ & $\begin{array}{l}\text { \% de estudiantes } \\
\text { en nivel avanzado }\end{array}$ \\
\hline 2009 & 299,81 & $14,55 \%$ & $51,09 \%$ & $27,39 \%$ & $7,128 \%$ \\
2012 & 312,65 & $13,01 \%$ & $47,23 \%$ & $28,74 \%$ & $11,11 \%$ \\
2014 & 299,02 & $17,75 \%$ & $47,25 \%$ & $26,26 \%$ & $8,89 \%$ \\
2016 & 292,18 & $20,18 \%$ & $47,19 \%$ & $25,01 \%$ & $7,75 \%$ \\
\hline
\end{tabular}

Nota. La tabla muestra que los resultados de los estudiantes de grado $11^{\circ}$ evidencian un comportamiento irregular. Sin embargo, en rango de los últimos 4 años evaluados se presentó una disminución progresiva de los promedios históricos anuales del establecimiento educativo.

\section{Tabla 3}

Resultados históricos a nivel nacional prueba SABER de ciencias naturales grado $11^{\circ}$

\begin{tabular}{ll}
\hline Aplicación & Puntaje promedio \\
\hline 2011 & 44,15 \\
2012 & 44,61 \\
\hline
\end{tabular}




\begin{tabular}{ll}
2013 & 43,89 \\
2014 & 49 \\
2015 & 49,02 \\
2016 & 51,43 \\
2017 & 49,86 \\
2018 & 48,09 \\
2019 & 46,85 \\
\hline Promedio histórico & 47,43 \\
\hline
\end{tabular}

Nota. La tabla presenta los resultados producto del análisis de los resultados en el establecimiento educativo, de carácter oficial, localizado en el distrito trístico de Riohacha, capital del Departamento de La Guajira, el cual se aleja de esta realidad académica relacionada con los aprendizajes limitados en materia de competencias científicas en Colombia.

Esta institución educativa ha obtenido deficientes resultados en el área de Ciencias Naturales principalmente en los niveles educativos de la básica secundaria y media, por lo cual desarrolló el ejercicio de investigación mediante el desarrollo de evaluaciones internas desde el área de química con el objetivo principal de establecer las condiciones actuales de la incidencia de la práctica pedagógica en los aprendizajes de los estudiantes.

En las tablas 4 y 5 se muestran los resultados de las aplicaciones históricas Saber Icfes de la prueba de ciencias naturales de los estudiantes de grado $5^{\circ}$ y $9^{\circ}$ respectivamente, indicando los dos indicadores de las pruebas, el puntaje promedio de los estudiantes (medido en una escala de 100 a 500), que muestra un comportamiento irregular de aumento y disminución en ambos grados evaluados y la distribución de los estudiantes en los niveles de desempeño de la prueba. En ambos grados la mayoría de la población estudiantil se ubicó históricamente en los niveles de menor desempeño (insuficiente y mínimo), en promedio el $91 \%$ de estudiantes de grado $5^{\circ}$ y el $88 \%$ de grado noveno no fueron capaces de responder acertadamente las preguntas de menor complejidad de las pruebas de ciencias naturales; aprendizajes mucho más preocupantes que los registrados en los promedios nacionales de $61,11 \%$ y $64,56 \%$ respectivamente.

\section{Tabla 4}

Resultados históricos de la prueba SABER de ciencias naturales grado $5^{\circ}$ del EE

\begin{tabular}{llllll}
\hline \multirow{2}{*}{ Aplicación Puntaje } & \multicolumn{4}{c}{ Niveles de desempeño } \\
\cline { 3 - 6 } & & $\begin{array}{l}\% \text { de estudiantes } \\
\text { nivel insuficiente }\end{array}$ & $\begin{array}{rlll}\text { \% de estudiantes } \\
\text { en nivel mínimo }\end{array}$ & $\begin{array}{l}\text { nivel satisfactorio } \\
\text { nivel }\end{array}$ & $\begin{array}{l}\text { \% de estudiantes en } \\
\text { nivel avanzado }\end{array}$ \\
\hline 2009 & 249 & $40 \%$ & $49 \%$ & $11 \%$ & $0 \%$ \\
2012 & 239 & $47 \%$ & $46 \%$ & $6 \%$ & $1 \%$ \\
2014 & 261 & $28 \%$ & $62 \%$ & $9 \%$ & $1 \%$ \\
2016 & 229,67 & $23 \%$ & $68 \%$ & $8 \%$ & $1 \%$ \\
\hline
\end{tabular}




\section{Tabla 5}

Resultados históricos de la prueba SABER de ciencias naturales grado $9^{\circ}$ del EE

\begin{tabular}{llllll}
\hline Aplicación & Puntaje & $\begin{array}{c}\text { Niveles de desempeño } \\
\text { nivel insuficiente }\end{array}$ & $\begin{array}{c}\text { estudiantes estudiantes } \\
\text { en nivel mínimo }\end{array}$ & $\begin{array}{l}\text { nivel satisfactorio } \\
\text { nividiantes }\end{array}$ & $\begin{array}{c}\text { en de estudiantes en } \\
\text { nivel avanzado }\end{array}$ \\
\hline 2009 & 235 & $39 \%$ & $54 \%$ & $7 \%$ & $0 \%$ \\
2012 & 263 & $24 \%$ & $60 \%$ & $16 \%$ & $0 \%$ \\
2014 & 241 & $34 \%$ & $54 \%$ & $12 \%$ & $0 \%$ \\
2016 & 262 & $29 \%$ & $57 \%$ & $12 \%$ & $3 \%$ \\
\hline
\end{tabular}

Nota. De acuerdo con la información de las tablas 4 y 5 se puede afirmar que solo el $9 \%$ de los estudiantes de grado $5^{\circ}$ y el $12 \%$ de grado tercero en promedio presentaron un desempeño adecuado en las competencias exigidas para el área y el grado evaluado, entendiendo que de acuerdo al ICFES (2020) el nivel satisfactorio es el nivel esperado que todos, o la gran mayoría de los estudiantes, deberían alcanzar. Adicionalmente un $1 \%$ de los estudiantes de ambos grados presento un desempeño avanzado, relacionado con resultados sobresalientes en las competencias esperadas para el área y el grado evaluados.

En cuanto al desarrollo de las competencias científicas evaluadas en las pruebas ICFES SABER del área de ciencias naturales para los grados $5^{\circ}$ y $9^{\circ}$, las tablas 6 y 7 muestran el comparativo anual del estado de estas competencias en comparación con los resultados de establecimientos educativos con puntaje promedio similares a los de la institución educativa IPC. Se evidencia que en ambos grados la competencia que presentó resultados más débiles fue la competencia de indagación, y la de uso comprensivo del conocimiento científico ha ido progresivamente debilitándose de acuerdo a los resultados obtenidos. La competencia de explicación de fenómenos presentó progresivo fortalecimiento de acuerdo al desempeño de los estudiantes.

\section{Tabla 6}

Comparativo del estado de las competencias con otros establecimientos con puntajes similares de estudiantes del grado $5^{\circ}$

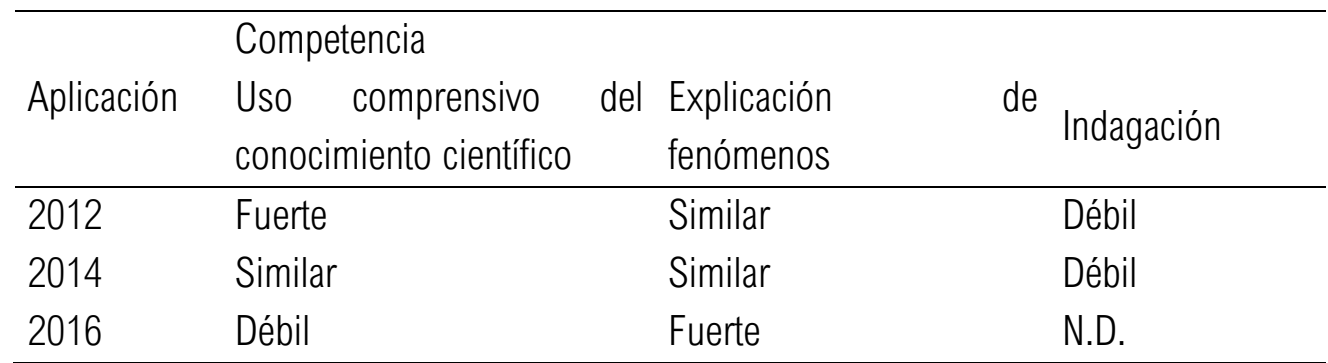




\section{Tabla 7}

Comparativo del estado de las competencias con otros establecimientos con puntajes similares de estudiantes del grado $9^{\circ}$

\begin{tabular}{cccc}
\hline \multirow{2}{*}{ Aplicación } & \multicolumn{3}{c}{ Competencia } \\
\cline { 2 - 4 } & $\begin{array}{c}\text { Uso comprensivo del } \\
\text { conocimiento científico }\end{array}$ & Explicación de fenómenos & Indagación \\
\hline 2012 & Fuerte & Fuerte & Débil \\
2014 & Fuerte & Similar & Débil \\
2016 & Similar & Muy fuerte & N.D. \\
\hline
\end{tabular}

Nota. En lo concerniente a los estudiantes de grado $11^{\circ}$, las tablas referenciadas presentan los puntajes históricos de las aplicaciones de las pruebas de ciencias naturales. Cabe destacar que las pruebas de 2011, 2012 y 2013 se aplicaron, de manera diferenciada, preguntas de las tres ciencias naturales (biología, química y física), cuyo resultado corresponde al promedio de estos tres puntajes. Los puntajes de los estudiantes del establecimiento educativo se mantuvieron por debajo del promedio nacional a excepción del año 2018, en el que registró 48,58 puntos frente al promedio nacional de 48,09 puntos.

\section{Tabla 8}

Resultados históricos de la prueba SABER de ciencias naturales grado $11^{\circ}$ del EE

\begin{tabular}{cccccc}
\hline \multirow{2}{*}{ Aplicación } & Puntaje & \multicolumn{4}{c}{ Distribución en los niveles de desempeño } \\
\cline { 3 - 6 } & & 1 & 2 & 3 & 4 \\
\hline 2011 & 40,17 & N.D. & N.D. & N.D. & N.D. \\
2012 & 42,39 & N.D. & N.D. & N.D. & N.D. \\
2013 & 43,84 & N.D. & N.D. & N.D. & N.D. \\
2014 & 45,4 & N.D. & N.D. & N.D. & N.D. \\
2015 & 46,51 & N.D. & N.D. & N.D. & N.D. \\
2016 & 50,14 & $8 \%$ & $73 \%$ & $19 \%$ & $0 \%$ \\
2017 & 48,45 & $16 \%$ & $68 \%$ & $16 \%$ & $0 \%$ \\
2018 & 48,58 & $17 \%$ & $64 \%$ & $19 \%$ & $0 \%$ \\
2019 & 46 & $28 \%$ & $57 \%$ & $15 \%$ & $0 \%$ \\
\hline Promedios históricos & 45,72 & $17 \%$ & $66 \%$ & $17 \%$ & $0 \%$ \\
\hline
\end{tabular}

Nota. De forma general, los niveles indican que ningún estudiante del grado $11^{\circ}$ del establecimiento educativo se ubicó en el nivel 4, relacionado con la capacidad y preparación para deducir y combinar procedimientos para realizar las tareas solicitadas en la prueba, mientras que la mayoría se ha ubicado históricamente en el nivel 2 y nivel 1 a solicitada (ICFES, 2020). 
En síntesis, los resultados históricos de la prueba SABER $11^{\circ}$ de los estudiantes demuestran mejoras en sus aprendizajes en relación con la capacidad de utilizar algunas habilidades de pensamiento y de procedimiento para evaluar predicciones (procesos químicos) y para derivar conclusiones para algunos fenómenos de la naturaleza basándose en conocimientos científicos y en la evidencia de su propia investigación y de la de otros (procesos físicos), mientras que los aprendizajes críticos, históricamente evidenciados, se relacionan con la deficiente capacidad de asociar fenómenos naturales con conceptos propios del conocimiento científico (procesos vivos, químicos y físicos).

Cabe destacar que los aprendices presentan marcadas limitaciones para establecer y diferenciar las competencias para poner en juego sus conocimientos básicos de las Ciencias Naturales en la comprensión y resolución de problemas (ICFES 2020), en el sentido de presentar una limitada comprensión sobre las particularidades y los alcances del conocimiento científico y la capacidad que poseen para diferenciar este conocimiento de otros saberes.

La propuesta de investigación desarrolló con el propósito de establecer, mediante la evaluación interna, desde el quehacer docente del área de ciencias naturales y educación ambiental del establecimiento educativo, las condiciones de desarrollo de las competencias científicas objetivo propuestas por el sistema educativo colombiano y evaluadas por el ICFES, a fin de tener un marco de referencia adicional a la vista internacional, nacional y regional provista por las evaluaciones referenciadas. Es decir, se trata de un esfuerzo por disponer desde la perspectiva de los principales protagonistas de la gestión educativa institucional, de acciones de evaluación y valoración del aporte del currículo institucional al desarrollo de las competencias científicas de los estudiantes de los niveles de educación básica secundaria y media.

\section{MÉTODO}

\section{Tipo de investigación}

La investigación se enmarca en el enfoque cuantitativo, de tipo no experimental, transeccional, iguiendo un diseño de tipo descriptivo, de de acuerdo a Hernández Sampieri et al. (2010), este tipo de investigaciones se caracterizan porque la meta del investigador consiste en describir fenómenos, situaciones, contextos y eventos, esto es, detallar cómo son y cómo se manifiestan.

\section{Diseño de la investigación}

La investigación posee un diseño no experimental, que según Hernández Sampieri et al. (2010), no requiere la manipulación deliberada de las variables por el investigador, al permitir explorar el fenómeno en su ámbito natural para luego analizarlo, más el investigador no posee el control total sobre las variables objeto de estudio. Se trató del diseño y aplicación única de un test, tipo cuestionario, de preguntas estandarizadas de lápiz y papel, de selección múltiple con única respuesta, discriminados por las competencias evaluadas, y derivadas de las preguntas liberadas del ICFES en sus aplicaciones históricas. 


\section{Población y muestra}

La población de la investigación la constituyen todos los estudiantes de educación básica secundaria y media vocacional de las instituciones educativas oficiales del Distrito de Riohacha, en el departamento de La Guajira.

La muestra estuvo constituida por 523 estudiantes que comprenden la población estudiantil de los niveles de educación básica secundaria y media vocacional de la sede central de la institución educativa durante la vigencia lectiva 2015.

Los criterios de selección de la muestra fueron los siguientes:

-Estudiantes matriculados en los grados de educación básica primaria, secundaria y media en la sede central del establecimiento educativo.

-Estudiantes cuyos acudientes firmaron el consentimiento informado.

-Estudiantes que hayan participado en la aplicación de los instrumentos de recolección de información.

En la tabla 7 se presenta la discriminación de la muestra de la investigación por niveles educativos y grados.

\section{Tabla 7}

Muestra del estudio

\begin{tabular}{|c|c|c|c|c|}
\hline Nivel Educativo & \multicolumn{2}{|c|}{ Grado } & $\begin{array}{l}\text { No. de estudiantes } \\
\text { por cada grado }\end{array}$ & $\begin{array}{ll}\text { No. de total } \\
\text { estudiantes por } \\
\text { grado }\end{array}$ \\
\hline \multirow{15}{*}{ Básica Secundaria } & \multirow{5}{*}{$6^{\circ}$} & $601^{\circ}$ & 36 & \multirow{5}{*}{149} \\
\hline & & $602^{\circ}$ & 31 & \\
\hline & & $603^{\circ}$ & 28 & \\
\hline & & $604^{\circ}$ & 26 & \\
\hline & & $605^{\circ}$ & 28 & \\
\hline & \multirow{5}{*}{$7^{\circ}$} & $701^{\circ}$ & 30 & \multirow{5}{*}{132} \\
\hline & & $702^{\circ}$ & 29 & \\
\hline & & $703^{\circ}$ & 27 & \\
\hline & & $704^{\circ}$ & 24 & \\
\hline & & $705^{\circ}$ & 22 & \\
\hline & \multirow{3}{*}{$8^{\circ}$} & $801^{\circ}$ & 32 & \multirow{3}{*}{99} \\
\hline & & $802^{\circ}$ & 34 & \\
\hline & & $803^{\circ}$ & 33 & \\
\hline & \multirow{2}{*}{$9^{\circ}$} & $901^{\circ}$ & 29 & \multirow{2}{*}{53} \\
\hline & & $902^{\circ}$ & 24 & \\
\hline \multirow{2}{*}{ Media vocacional } & \multirow{2}{*}{$10^{\circ}$} & $1001^{\circ}$ & 31 & \multirow{2}{*}{57} \\
\hline & & $1002^{\circ}$ & 26 & \\
\hline
\end{tabular}




\begin{tabular}{lll}
\hline \multicolumn{1}{c}{$11^{\circ}$} & $1101^{\circ}$ & 18 \\
& $1102^{\circ}$ & 15 \\
\hline No. total de estudiantes de educación básica & 433 Estudiantes \\
No. total de estudiantes de educación media vocacional & 90 Estudiantes \\
No. total de estudiantes (educación básica y media) & 523 Estudiantes \\
\hline
\end{tabular}

Nota. Muestra de studio abarcada por la investigación.

\section{Técnicas e instrumentos de recolección de datos}

La investigación utilizó un instrumento para la recolección de la información, se utilizó una rejilla de evaluación de las competencias científicas de los estudiantes a partir de la aplicación de los test, tipo cuestionarios de selección múltiple con única respuesta, diseñados de manera discriminadas por cada una de las competencias científicas evaluadas en Colombia (uso comprensivo del conocimiento científico, explicación de fenómenos e indagación).

La rejilla tuvo en cuenta las dimensiones de la variable de estudio: competencias científicas, a partir del establecimiento de indicadores, a los cuales se les otorga una valoración posible de 100 puntos, por lo que la valoración mínima fue de 0 (0\%) y la máxima fue de 10 (100\%). A partir de estas valoraciones se establecieron 4 posibles niveles de desempeño: insuficiente (valoración entre 0 y 3 , correspondiente a un $0 \%$ y 30\%), mínimo (valoración entre 4 y 6, equivalente al rango de 40\%-60\%), satisfactorio (valoración entre 7 y 8, 70\%-80\%) y avanzado (valoración entre 9 y 10, 90\%-100\%).

La rejilla de evaluación fue validada mediante juicio de expertos, esto es, cinco docentes de la línea de investigación en enseñanza de la química de la Universidad del Zulia. Adicionalmente, se realizó una prueba piloto con niños de características similares a los de la muestra, a partir de los resultados obtenidos se efectuaron los ajustes pertinentes a los instrumentos, cuyos resultados presentan similitud con los hallazgos de las pruebas estandarizadas aplicadas históricamente en la institución y en el país en el área de ciencias naturales.

Los instrumentos previamente ajustados se aplicaron a los estudiantes.

\section{Técnicas de análisis de datos}

El análisis e interpretación de los datos obtenidos a partir de la aplicación de las técnicas e instrumentos de investigación se basó en el tratamiento mediante estadística descriptiva a través de tablas de frecuencia para datos agrupados, de acuerdo a debe considerar la codificación física de los datos, su organización mediante una matriz como documento de un programa estadístico (para el caso particular del presente estudio se utilizó el programa excel), mediante su ejecución, exploración de los datos registrados y tratamiento estadístico (distribución de frecuencia, porcentajes válidos y acumulados, distribuciones gráficas, medidas de tendencia central y medidas de variabilidad) con base en los objetivos y las variables de estudio (Hernández Sampieri et al., 2010). 


\section{RESULTADOS}

A continuación, se presenta la descripción de los niveles de adquisición y desarrollo de las competencias científicas, establecidas como objetivo de aprendizaje de acuerdo al sistema educativo por competencias colombiano, producto de la evaluación de las habilidades y capacidades conceptuales y metodológicas a través de pruebas estandarizadas de lápiz y papel.

Los resultados estadísticos de la aplicación del instrumento de evaluación de las pruebas estandarizadas de lápiz y papel de la investigación se presentan asimilando la estructura del Instituto de Evaluación de la Calidad de la Educación en Colombia -ICFES- discriminados por la distribución poblacional de los estudiantes evaluados en niveles de desempeño (insuficiente, mínimo, satisfactorio y avanzado), con el análisis complementario de las variables de estadística descriptiva como el promedio, la desviación estándar y la varianza.

\section{Competencia de uso comprensivo del conocimiento científico}

Teniendo en cuenta las características descritas de componentes de la práctica pedagógica del área de química del establecimiento educativo abordado, derivados de la investigación marco de la cual se desprende el presente artículo y publicado mediante otro escrito académico (Blanchar Añez, 2020), caracterizadas por su naturaleza tradicional y magistral, en las que se priorizan la memorización y repetición literal de la información.

De hecho, a partir del análisis estadístico de los datos para cada una de las competencias científicas evaluadas confirman esta suposición, los cuales se presentan en las siguientes gráficas: figura 1 (grado $6^{\circ}$ de EBS), figura 2 (grado $7^{\circ}$ de EBS), figura 3 (grado $8^{\circ}$ de EBS), figura 4 (grado $9^{\circ}$ de EBS), figura 5 (grado $10^{\circ}$ de EMV) y figura 6 (grado $11^{\circ}$ de EMV).

\section{Figura 1}

\section{Distribución estudiantil por niveles de desempeño de los estudiantes de grado $6^{\circ}$ de EBS, de acuerdo a la competencia científica Uso comprensivo del conocimiento científico}

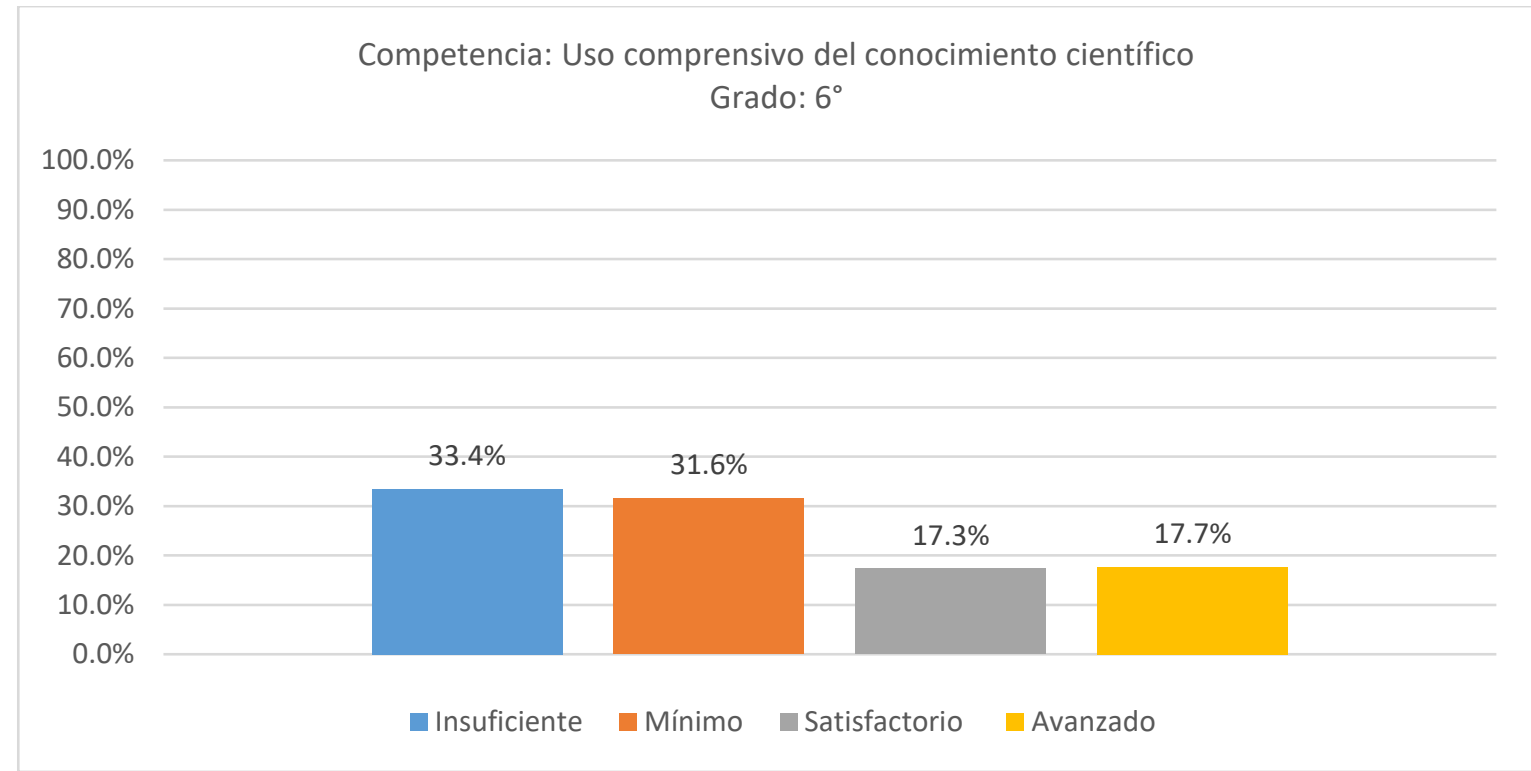




\section{Figura 2}

Distribución estudiantil por niveles de desempeño de los estudiantes de grado $7{ }^{\circ}$ de EBS, de acuerdo a la competencia cientifica Uso comprensivo del conocimiento cientifico

\section{Competencia: Uso comprensivo del conocimiento científico}

Grado: $7^{\circ}$

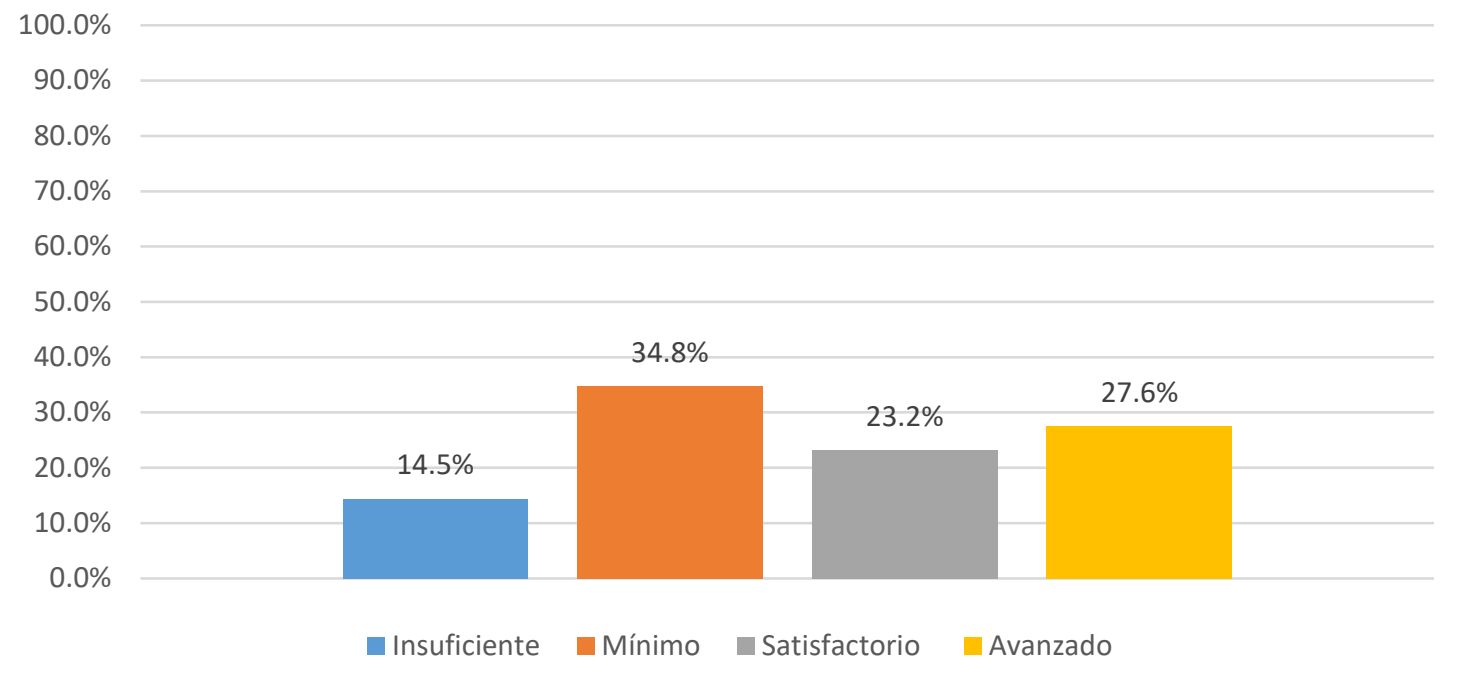

\section{Figura 3}

Distribución estudiantil por niveles de desempeño de los estudiantes de grado $8^{\circ}$ de EBS, de acuerdo a la competencia científica Uso comprensivo del conocimiento científico

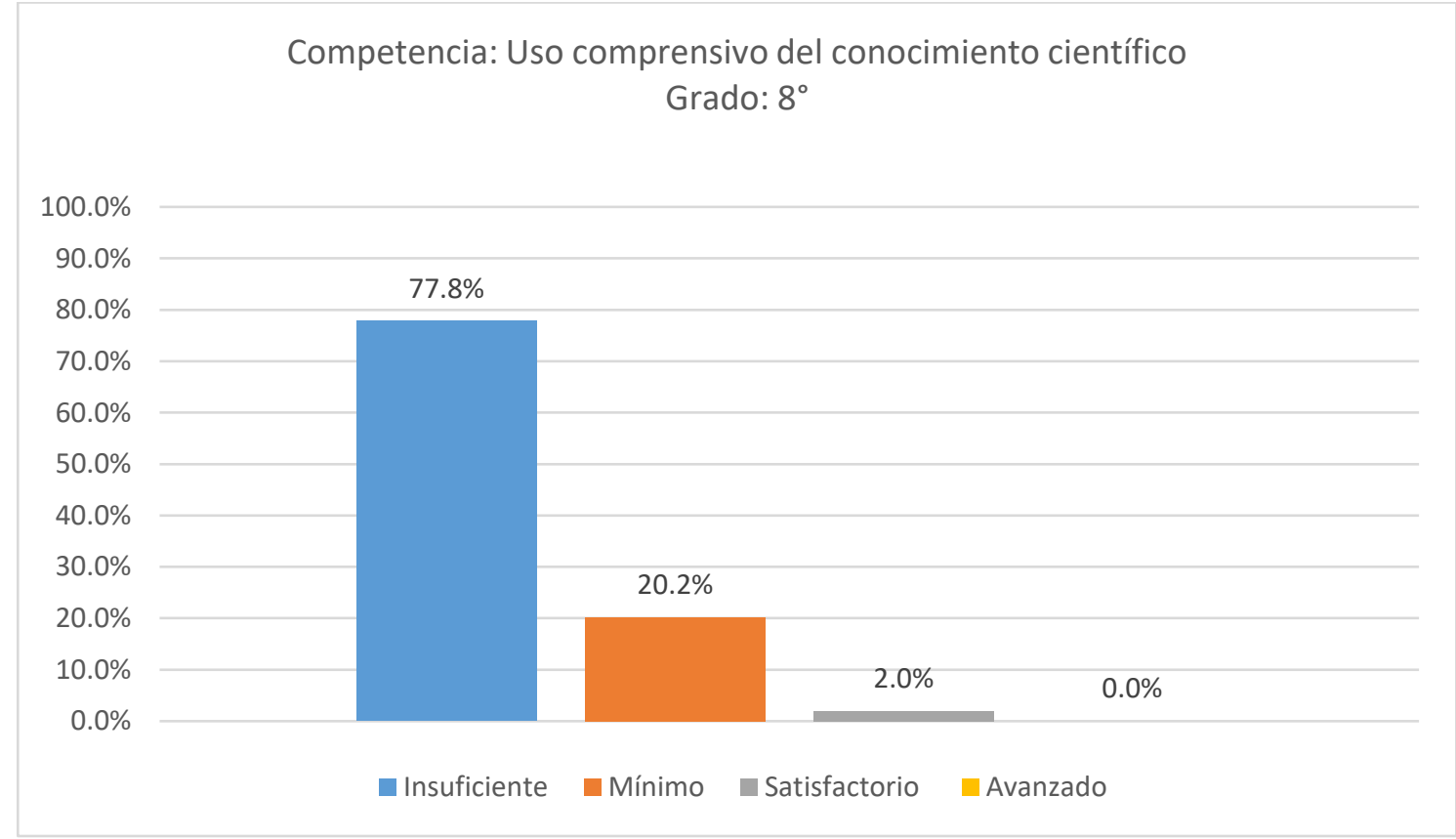




\section{Figura 4}

Distribución estudiantil por niveles de desempeño de los estudiantes de grado $9^{\circ}$ de EBS, de acuerdo a la competencia cientifica Uso comprensivo del conocimiento cientifico

\section{Competencia: Uso comprensivo del conocimiento científico \\ Grado: $9^{\circ}$}

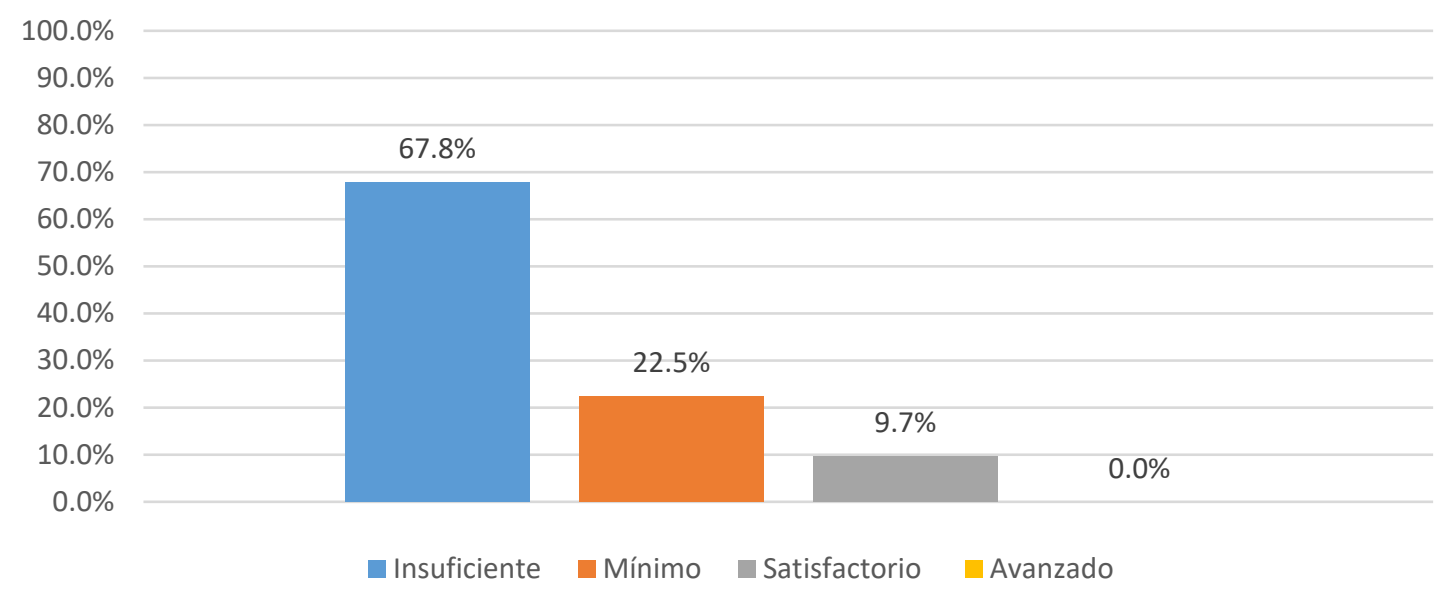

\section{Figura 5}

Distribución estudiantil por niveles de desempeño de los estudiantes de grado $10^{\circ}$ de EMV, de acuerdo a la competencia científica Uso comprensivo del conocimiento científico

Competencia: Uso comprensivo del conocimiento científico

Grado: $10^{\circ}$

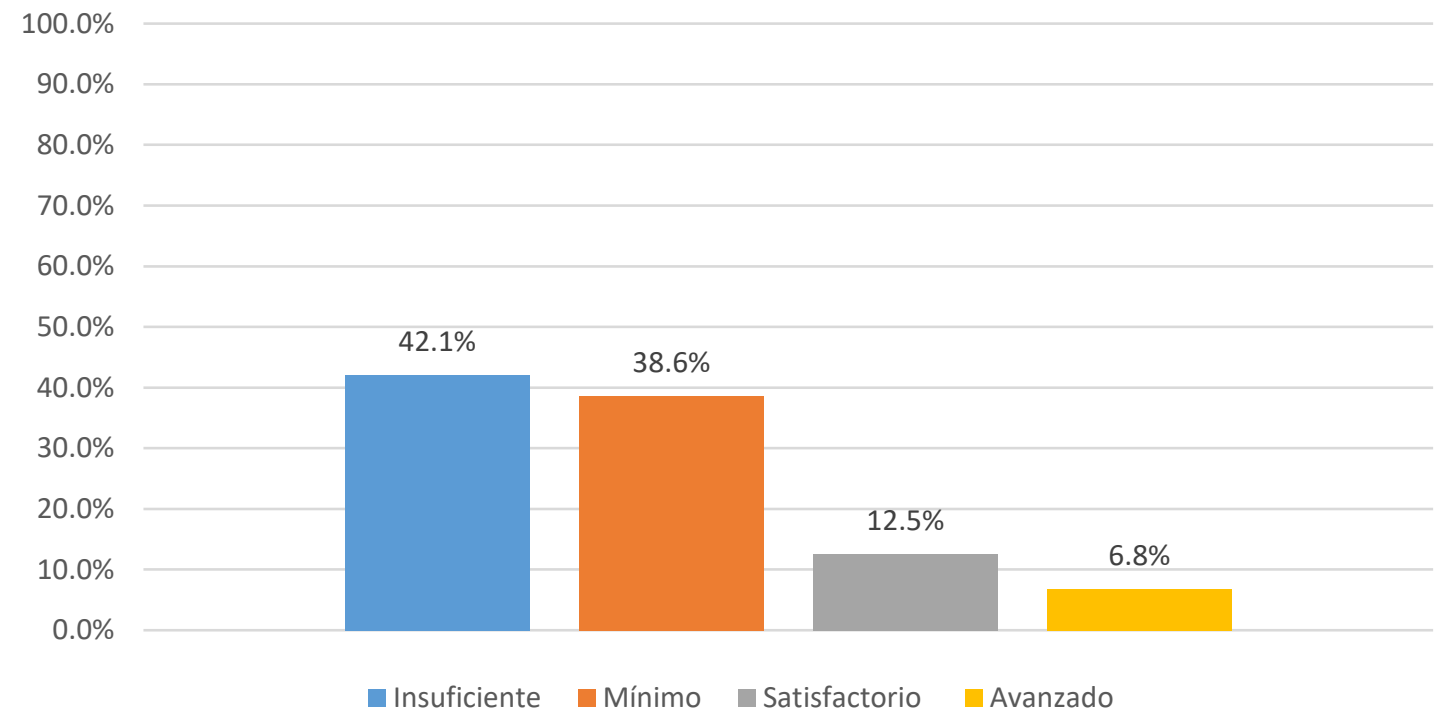




\section{Figura 6 \\ Distribución estudiantil por niveles de desempeño de los estudiantes de grado $11^{\circ}$ de EMV, de acuerdo a la competencia científica Uso comprensivo del conocimiento científico}

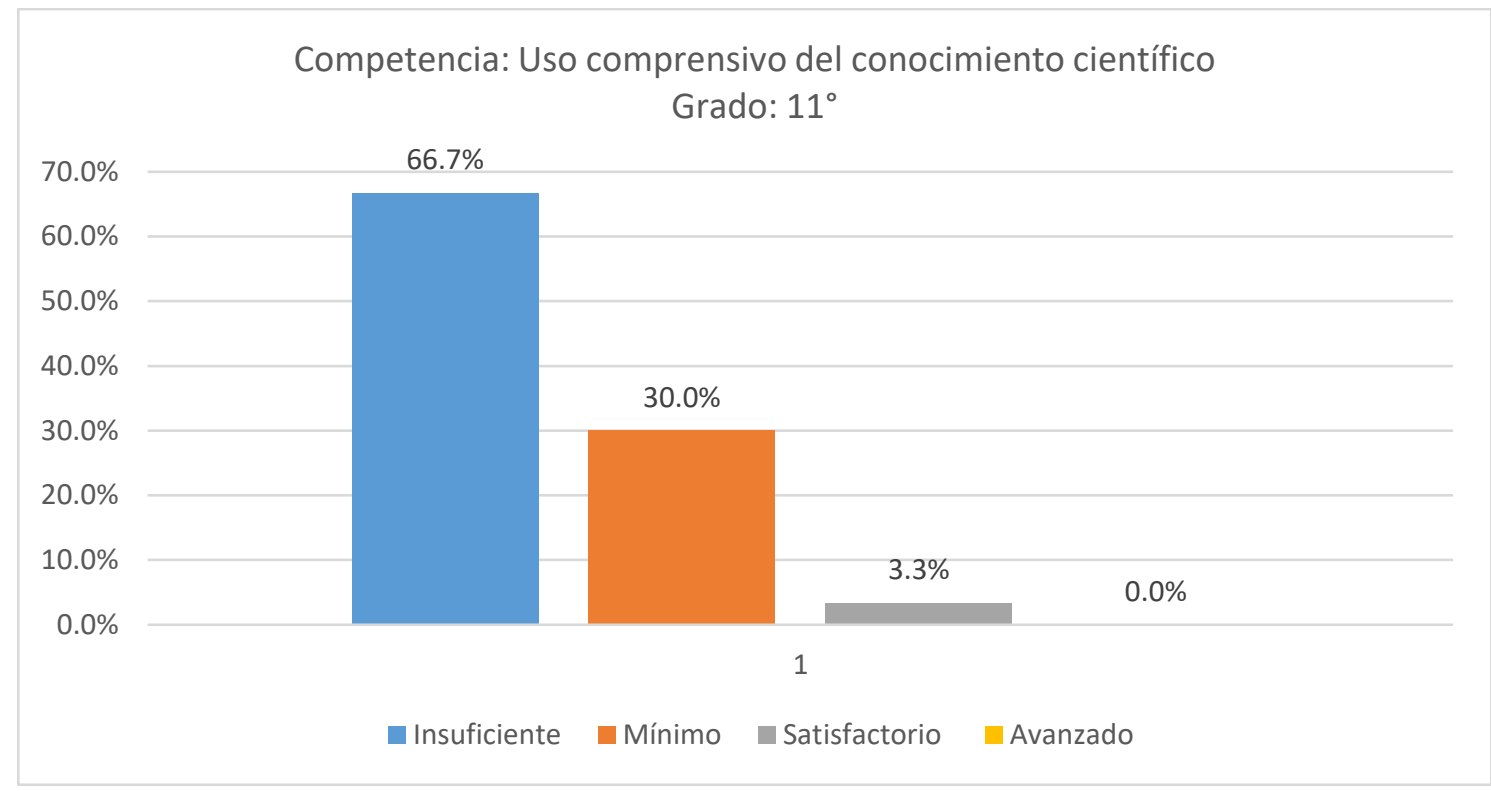

Nota. Se evidencia que en la competencia uso comprensivo del conocimiento científico se presentaron los mejores resultados de aprendizaje en comparación con las otras competencias evaluadas, sin embargo, es importante destacar el progresivo déficit de aprendizaje y desempeño estudiantil a medida que se avanza desde los grados inferiores hasta los superiores.

Esta situación significaría que las prácticas pedagógicas de enseñanza de ciencias naturales no propician de manera significativa el desarrollo las habilidades comprensión y uso de los conocimientos científicos de los estudiantes, la construcción de explicaciones y la capacidad de plantear preguntas y procedimientos científicos adecuados para dar respuesta a estos interrogantes.

De acuerdo con los resultados obtenidos la mayor población de los estudiantes con mejores resultados en la competencia de uso comprensivo del conocimiento científico se registró en los grados inferiores $\left(6^{\circ}\right.$ y $\left.7^{\circ}\right)$, con un $34,9 \%$ y un $50,8 \%$ en los mejores niveles de desempeño (satisfactorio y avanzado), mientras que los grados superiores registraron la menor población con desempeño satisfactorios, 2\% para el grado $8^{\circ}, 9,4 \%$ para el grado $9^{\circ}, 19,3 \%$ para el grado $10^{\circ}$ y $3 \%$ para el grado $11^{\circ}$.

Los desempeños de los estudiantes relacionados con esta competencia (mejores en comparación con las competencias de explicación e indagación) representan el resultado de la enseñanza y aprendizaje a partir de las directrices curriculares de la planeación, las prácticas de aula y la evaluación interna institucional en materia de contenidos declarativos 0 conceptuales, y en menor medida con actividades de análisis, explicación y/o comprobación de los fenómenos a través de la experimentación. 
En este sentido se puede evidenciar como se agudiza el desempeño estudiantil en el área de las ciencias naturales para poner en evidencia su capacidad de comprender y usar conceptos, teorías y modelos de las ciencias en la solución de problemas, teniendo en cuenta que el estudiante no repita de memoria los términos técnicos ni sus definiciones, sino que los comprenda y aplique en la resolución de problemas.

\section{Síntesis comparativa de los resultados de la competencia Uso comprensivo del conocimiento científico}

En la figura 7 se muestra la evolución progresiva de las dificultades y habilidades críticas de los estudiantes ante la competencia analizada desde los grados inferiores hasta los superiores, observándose como aumenta la población de los niveles de menor desempeño (barras de color azul y naranja) y disminuye la población ubicada en los niveles de desempeño superior (barras de color gris y amarillo) a medida que se transita por la educación básica y media secundaria.

\section{Figura 7}

Síntesis de la distribución porcentual de la población estudiantil en relación a la competencia de Uso comprensivo del conocimiento científico

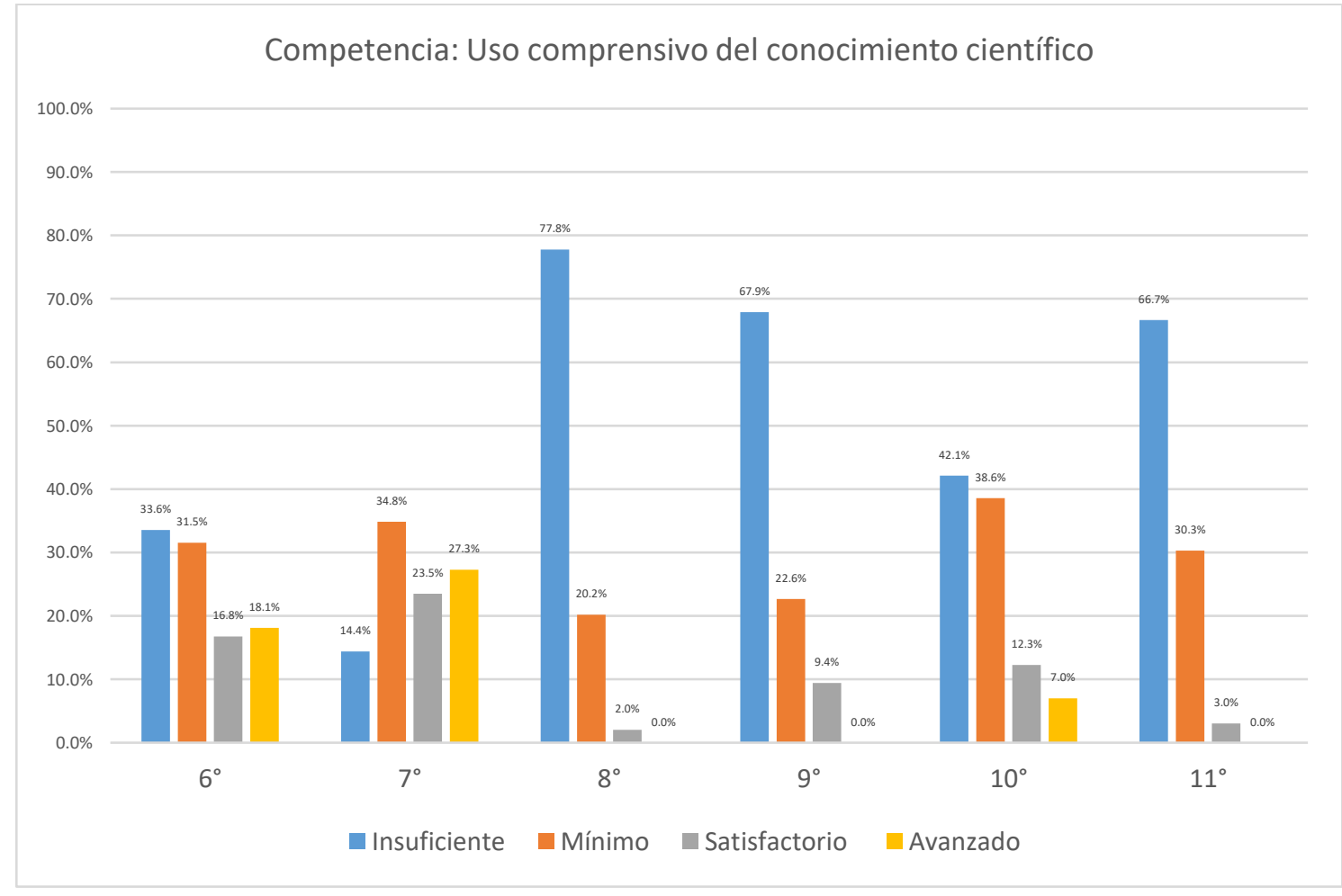

\section{Competencia científica Explicación de Fenómenos}

Los resultados de la evaluación de esta competencia científica evidenciaron un mayor nivel de criticidad de los aprendizajes de los estudiantes relacionados con la capacidad para construir explicaciones, así como para comprender argumentos y modelos que den razón de los fenómenos. Es decir que si se está generando un déficit progresivo de la habilidad para usar comprensivamente el conocimiento científico. Al igual que en la competencia anterior, de acuerdo a los resultados expuestos en la gráfica 3 se observa el progresivo déficit de 
aprendizaje y desempeño estudiantil a medida que se avanza desde los grados inferiores, pero con un deficiente desempeño estudiantil mucho más marcado, teniendo en cuenta la mayor población estudiantil ubicada en los grados inferiores de desempeño (barras azul y naranja en la figuras $8,9,10,11,12$ y 13).

\section{Figura 8}

Distribución estudiantil por niveles de desempeño de los estudiantes de grado $6^{\circ}$ de $E B S$, de acuerdo a la competencia científica Explicación de fenómenos

Competencia: Explicación de fenomenos

Grado: $6^{\circ}$

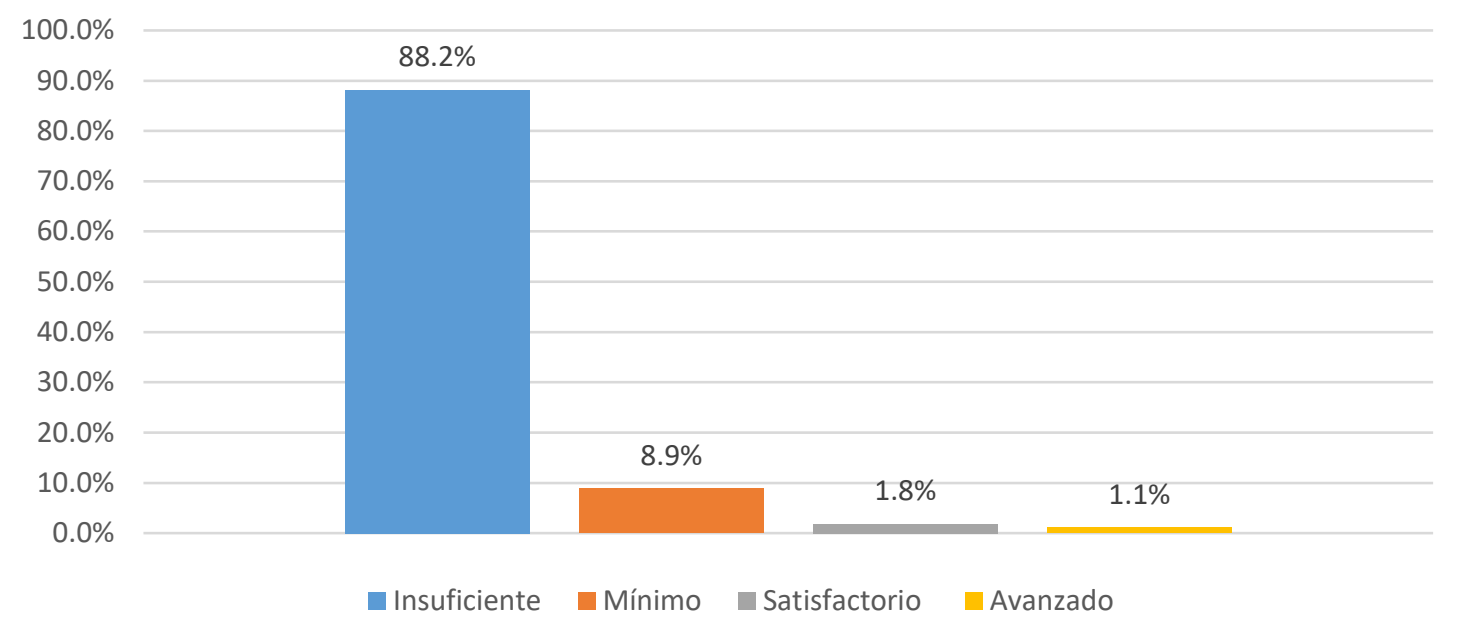

Figura 9

Distribución estudiantil por niveles de desempeño de los estudiantes de grado $7^{\circ}$ de EBS, de acuerdo a la competencia científica Explicación de fenómenos

Competencia: Explicación de fenomenos

Grado: $7^{\circ}$

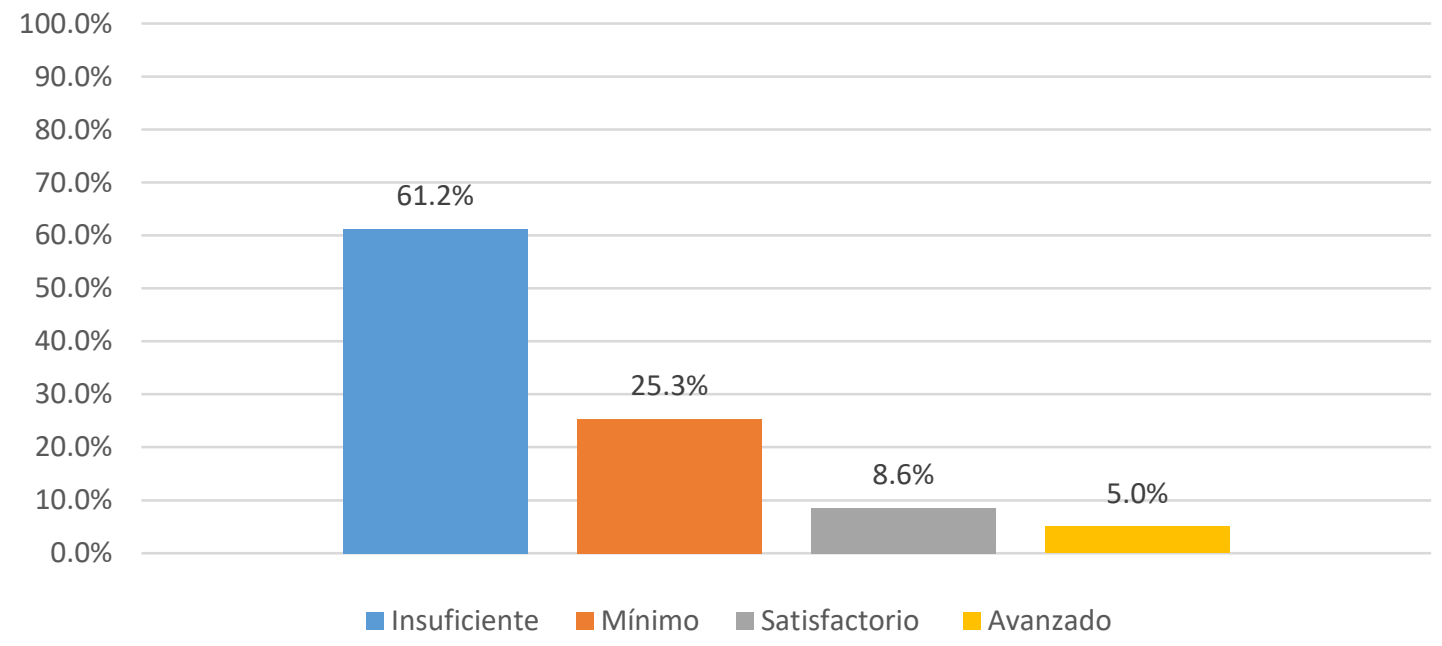




\section{Figura 10}

Distribución estudiantil por niveles de desempeño de los estudiantes de grado $8^{\circ}$ de EBS, de acuerdo a la competencia cientifica Explicación de fenómenos

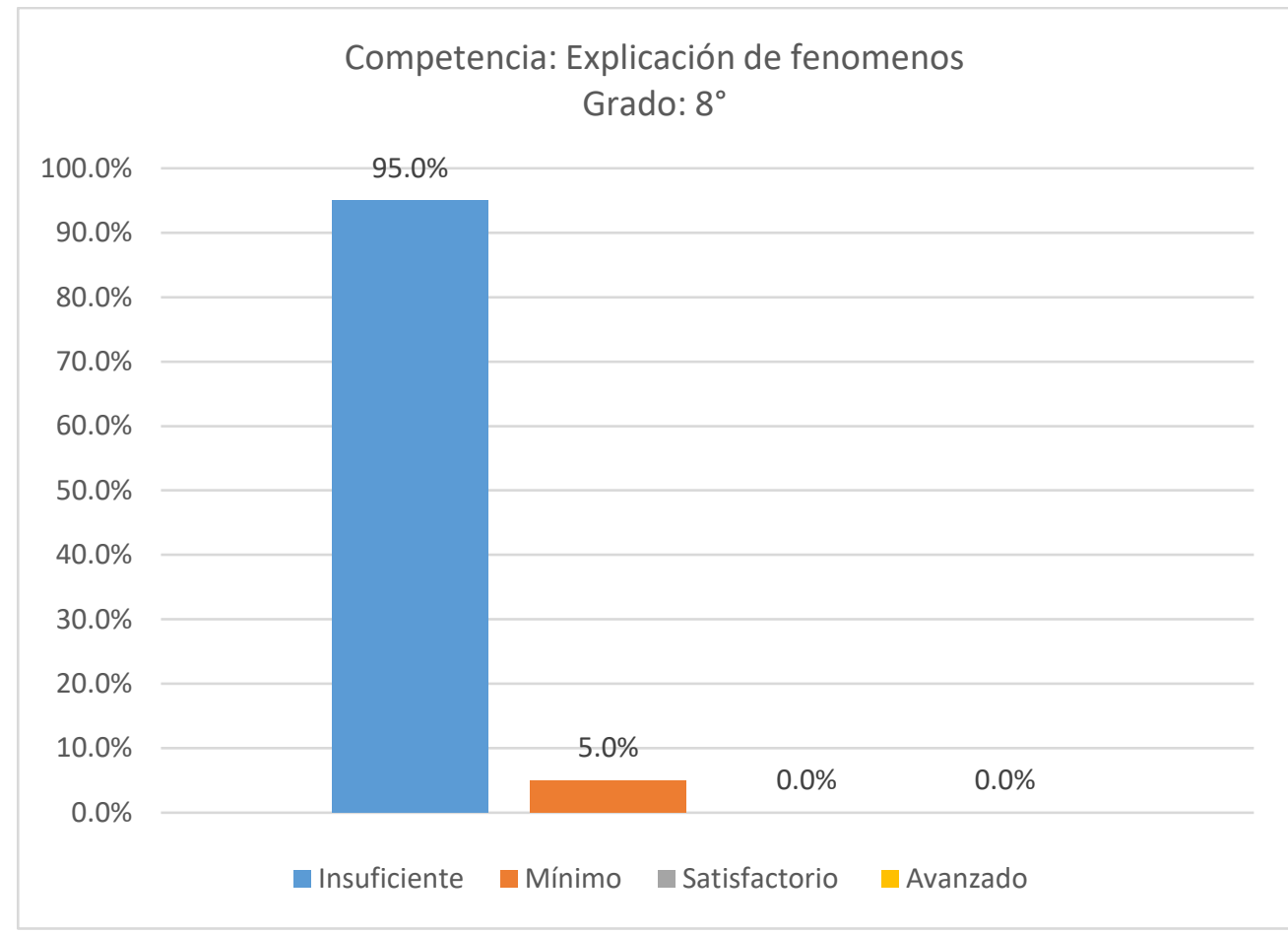

\section{Figura 11}

Distribución estudiantil por niveles de desempeño de los estudiantes de grado $9^{\circ}$ de EBS, de acuerdo a la competencia científica Explicación de fenómenos

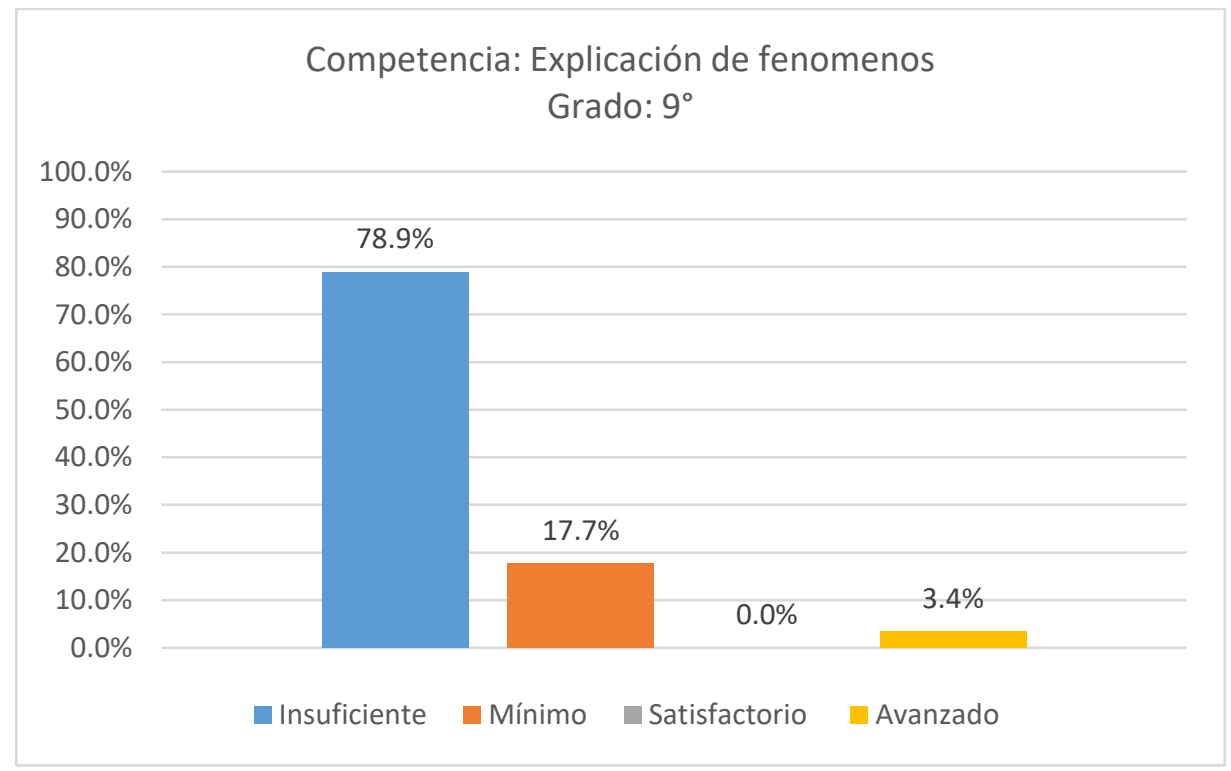




\section{Figura 12}

Distribución estudiantil por niveles de desempeño de los estudiantes de grado $10^{\circ}$ de EMV, de acuerdo a la competencia cientifica Explicación de fenómenos

\section{Competencia: Explicación de fenomenos Grado: $10^{\circ}$}

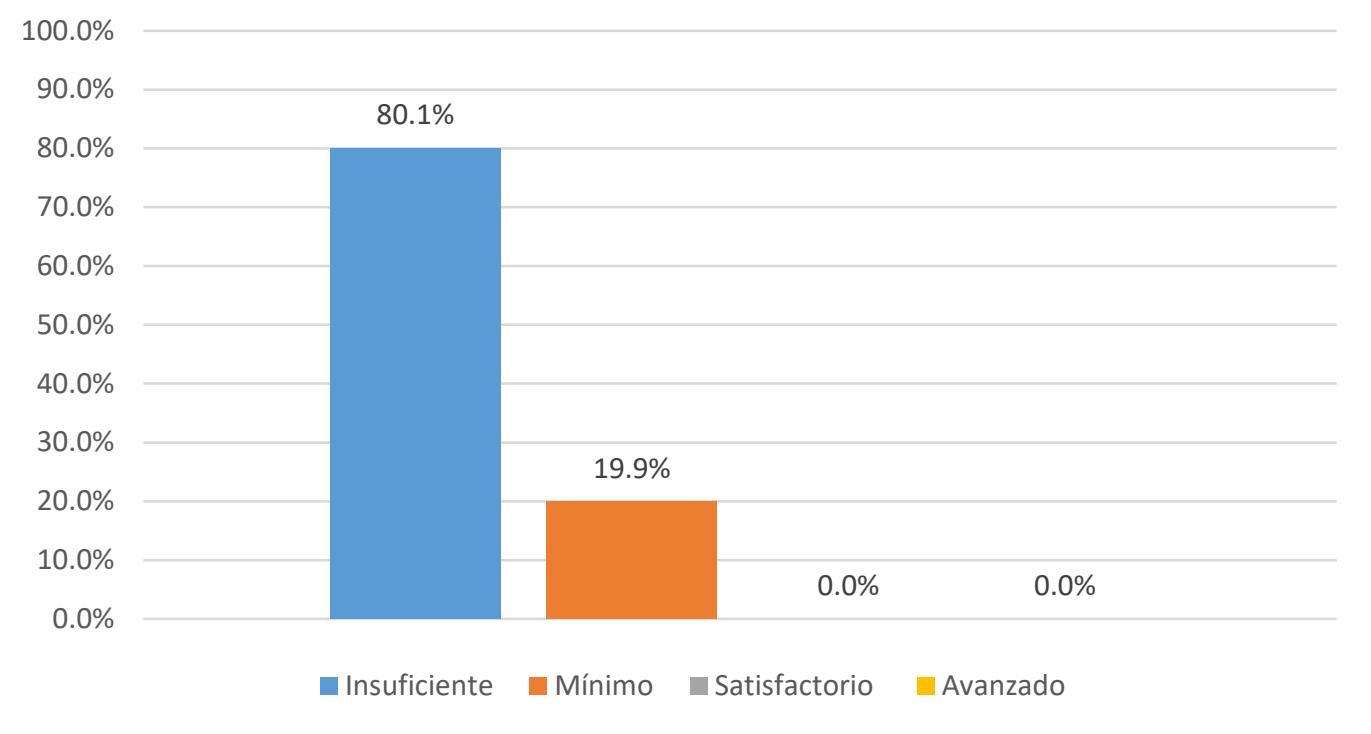

\section{Figura 13}

Distribución estudiantil por niveles de desempeño de los estudiantes de grado $11^{\circ}$ de EMV, de acuerdo a la competencia cientifica Explicación de fenómenos

\section{Competencia: Explicación de fenomenos Grado: $11^{\circ}$}

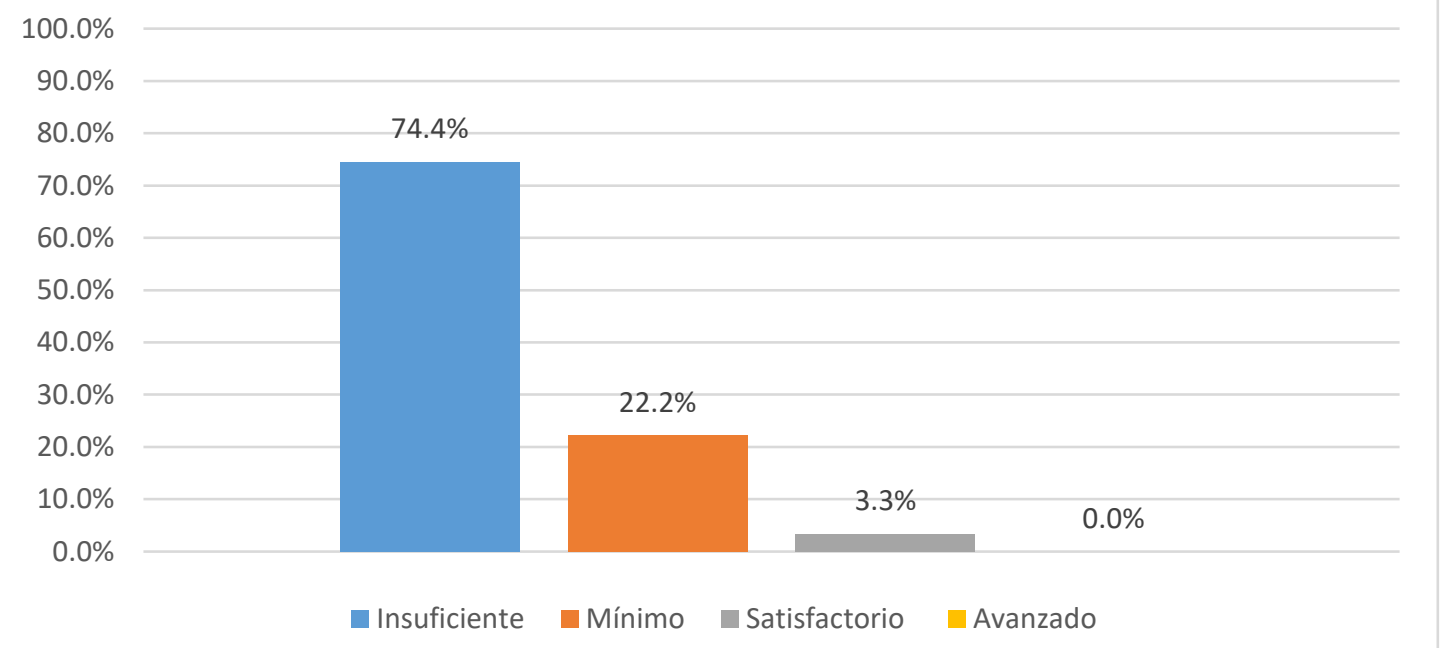

Nota. Tal cómo se describió en el ítem anterior para la competencia de uso comprensivo, el nivel de análisis y de actitud crítica requeridos en los estudiantes para establecer la validez o coherencia de una afirmación a través de esta segunda competencia, siendo de mayor exigencia y reto cognitivo, representa una mayor exigencia 
académica tanto para los docentes del área como para los estudiantes. Y de hecho así lo demuestran las evidencias de la aplicación del instrumento de evaluación.

La mayor población de los estudiantes con mejor desempeño en la competencia de expiación de fenómenos (niveles satisfactorio y avanzado) se presenta en los grados inferiores, específicamente en el grado $7^{\circ}$ con un $13,6 \%$, el grado $9^{\circ}$ con un $3,8 \%$, seguido del grado $6^{\circ}$ con un $3,3 \%$ y el grado $11^{\circ}$ con un $3 \%$, mientras que en los grados $8^{\circ}, 10$ y $11^{\circ}$ ninguno de los estudiantes evaluados se ubicó en estos niveles de desempeño.

Por su parte, en la figura 14 se muestra la mayoritaria población estudiantil ubicada los niveles de menor desempeño (barras de color azul y naranja) y la disminución de la población ubicada en los niveles de desempeño superior (barras de color gris y amarillo) en referencia con la competencia para la explicación de fenómenos, en mayor medida que los resultados identificados para la competencia de uso comprensivo del conocimiento científico.

\section{Figura 14}

Síntesis de la distribución porcentual de la población estudiantil en relación a la competencia de Explicación de Fenómenos

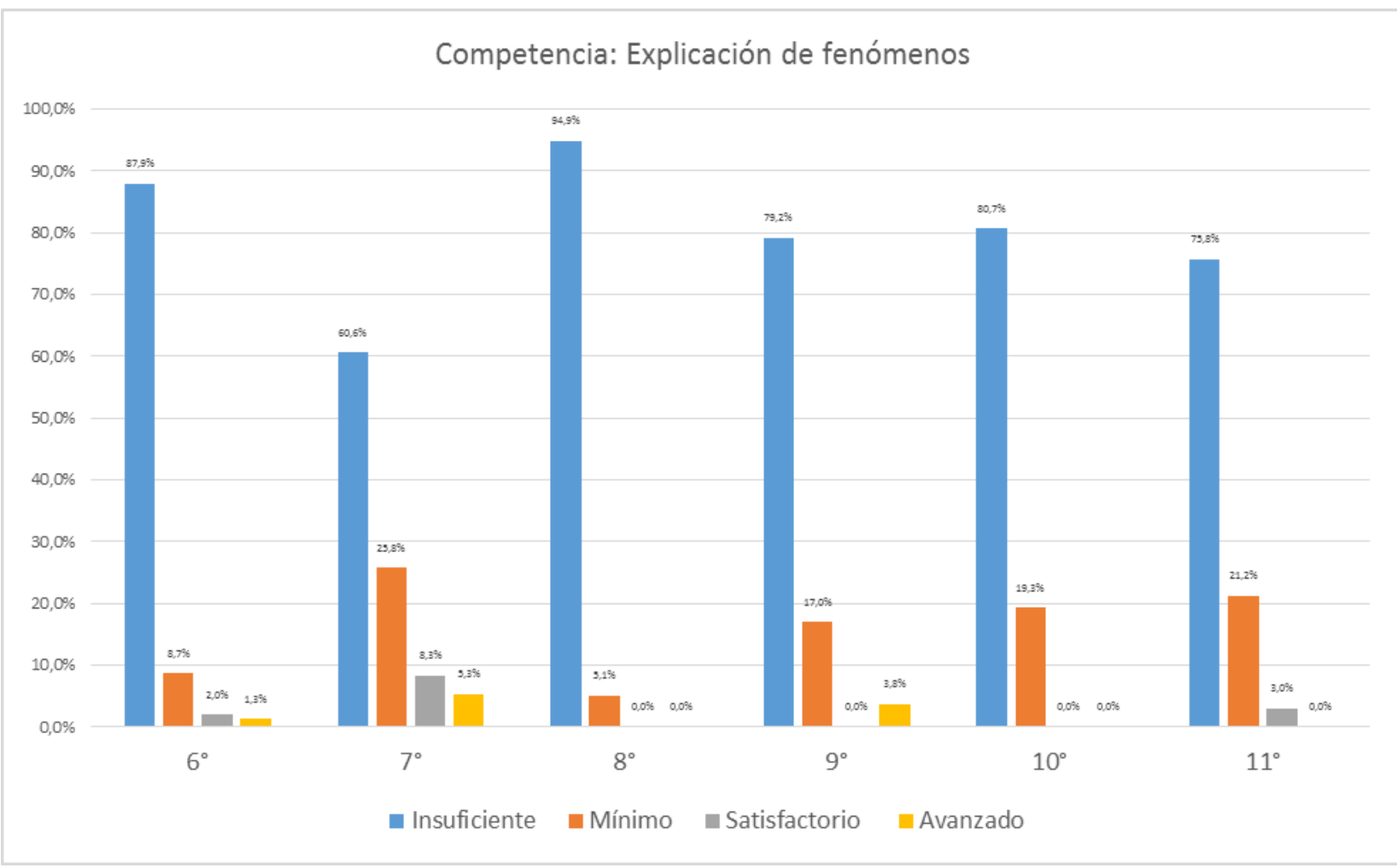

\section{Competencia científica Indagación}

En los resultados relacionados con la capacidad para plantear preguntas y procedimientos adecuados, así como para buscar, seleccionar, organizar e interpretar información relevante para dar respuesta a esos interrogantes, aspectos que caracterizan a la competencia de indagación, la tendencia no difiere de los resultados detectados 
en la competencia de explicación de fenómenos, en ambas la población estudiantil se ubicó mayoritariamente en los niveles de desempeño inferiores. Esta información se presenta en las figuras 15, 16, 17, 18, 19 y 20.

\section{Figura 15}

Distribución estudiantil por niveles de desempeño de los estudiantes de grado $6^{\circ}$ de $E B S$, de acuerdo a la competencia científica Indagación

\section{Competencia: Indagación}

Grado: $6^{\circ}$

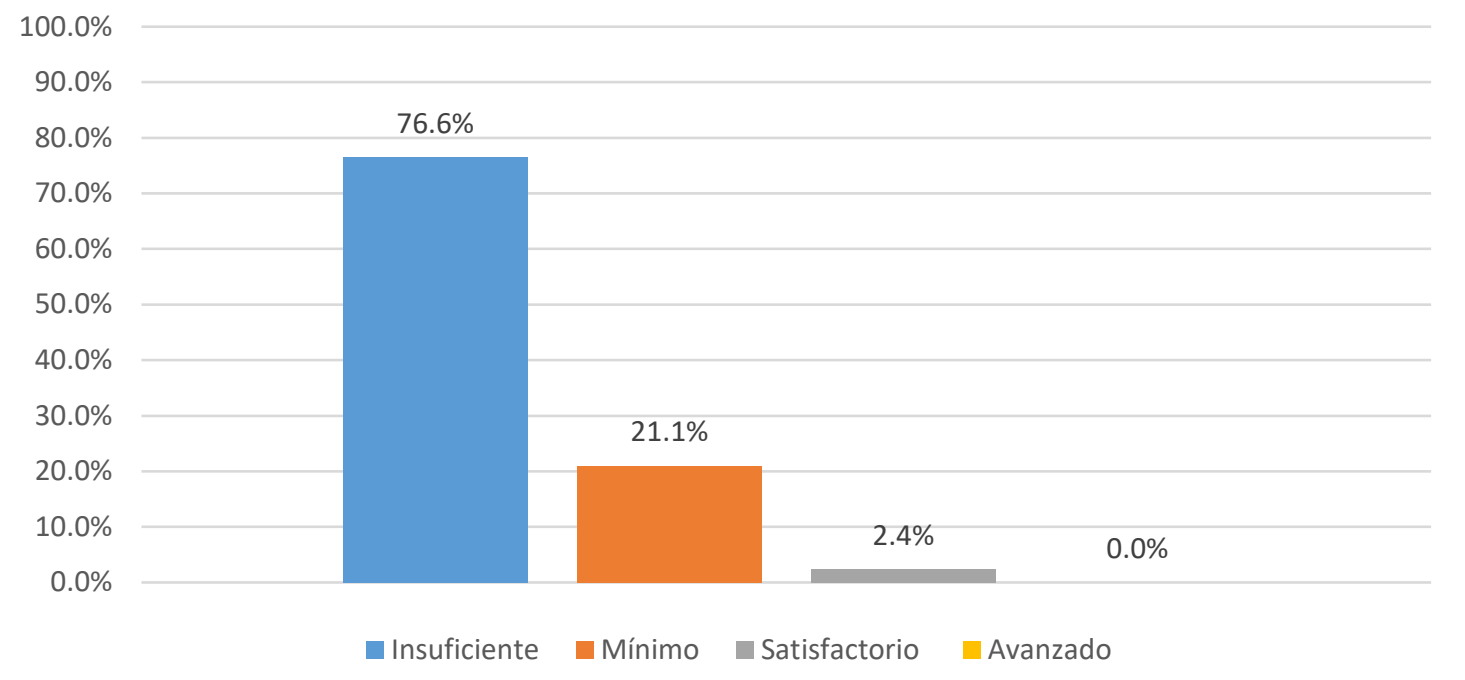




\section{Figura 16}

Distribución estudiantil por niveles de desempeño de los estudiantes de grado $8^{\circ}$ de EBS, de acuerdo a la competencia cientifica Indagación

\section{Competencia: Indagación \\ Grado: $7^{\circ}$}

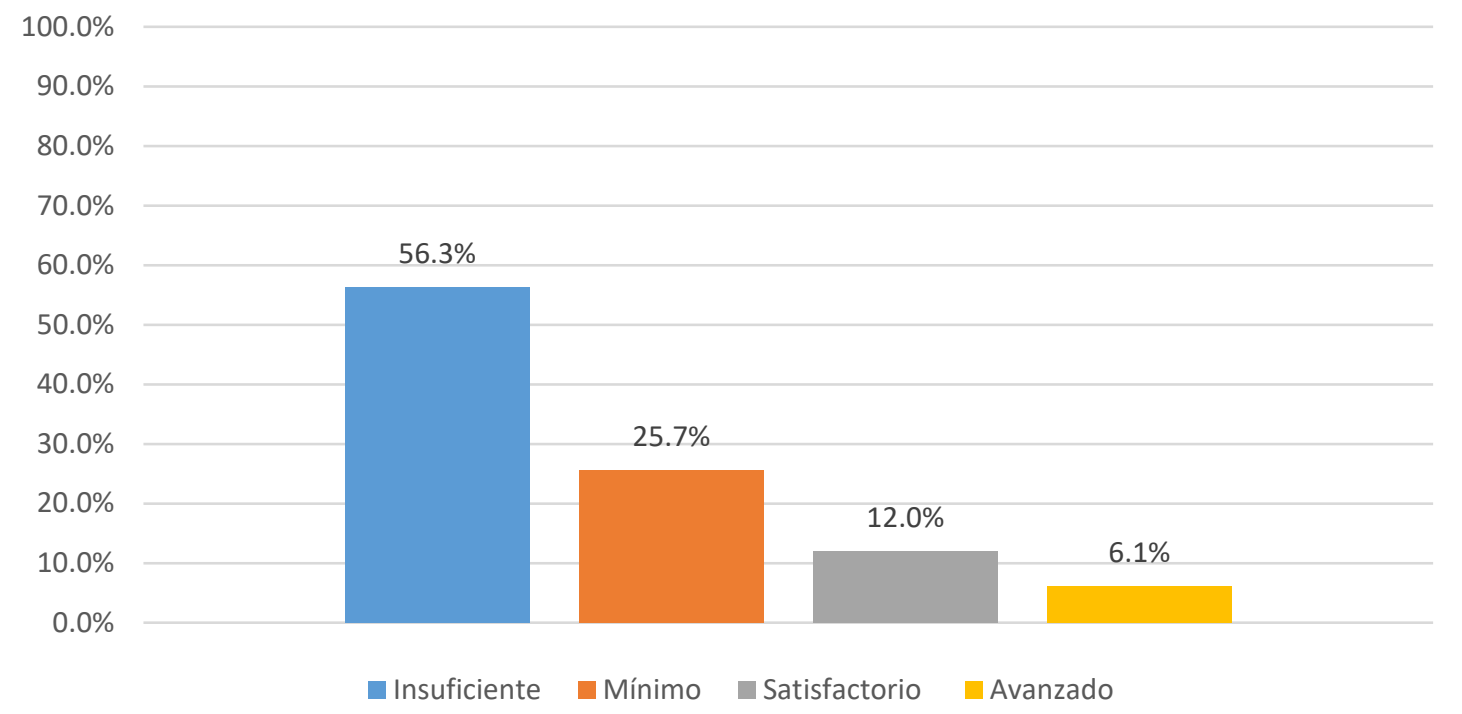

\section{Figura 17}

Distribución estudiantil por niveles de desempeño de los estudiantes de grado $8^{\circ}$ de EBS, de acuerdo a la competencia científica Indagación

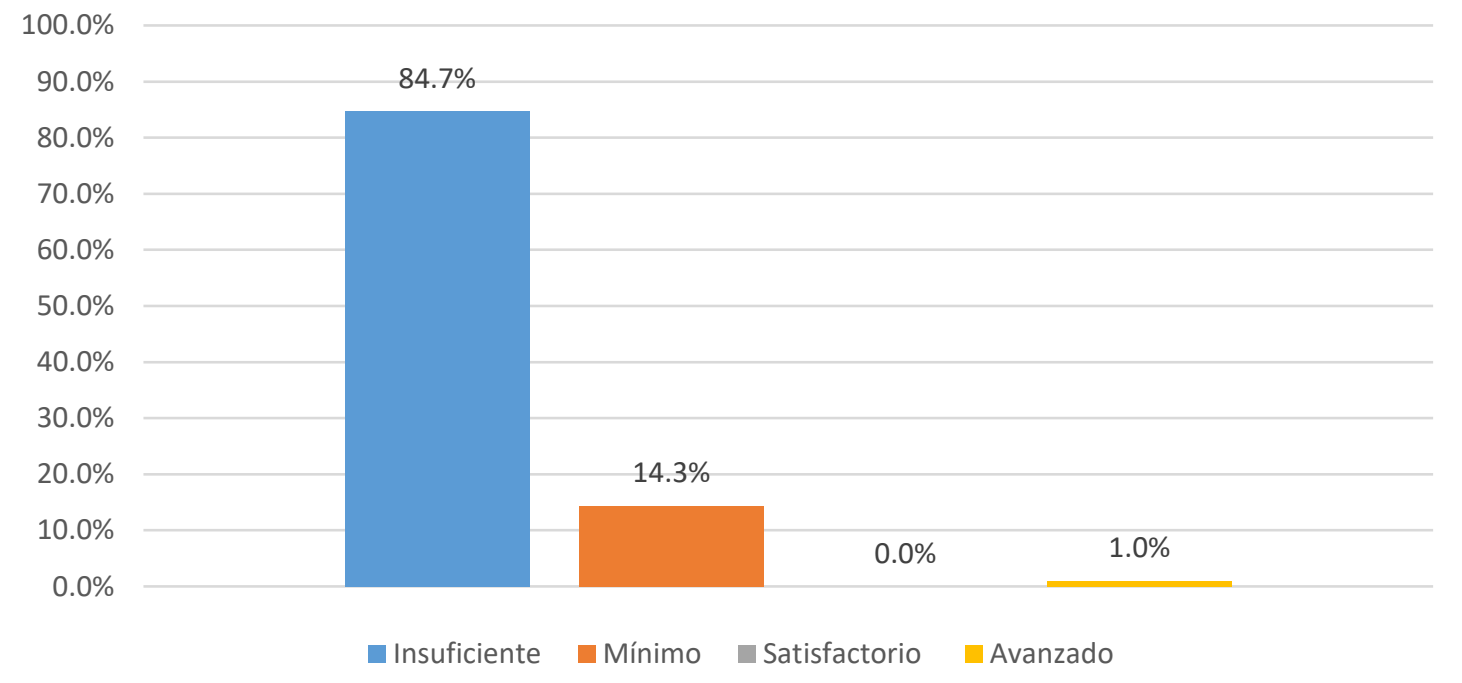




\section{Figura 18}

Distribución estudiantil por niveles de desempeño de los estudiantes de grado $9^{\circ}$ de EBS, de acuerdo a la competencia cientifica Indagación

\section{Competencia: Indagación \\ Grado: $9^{\circ}$}

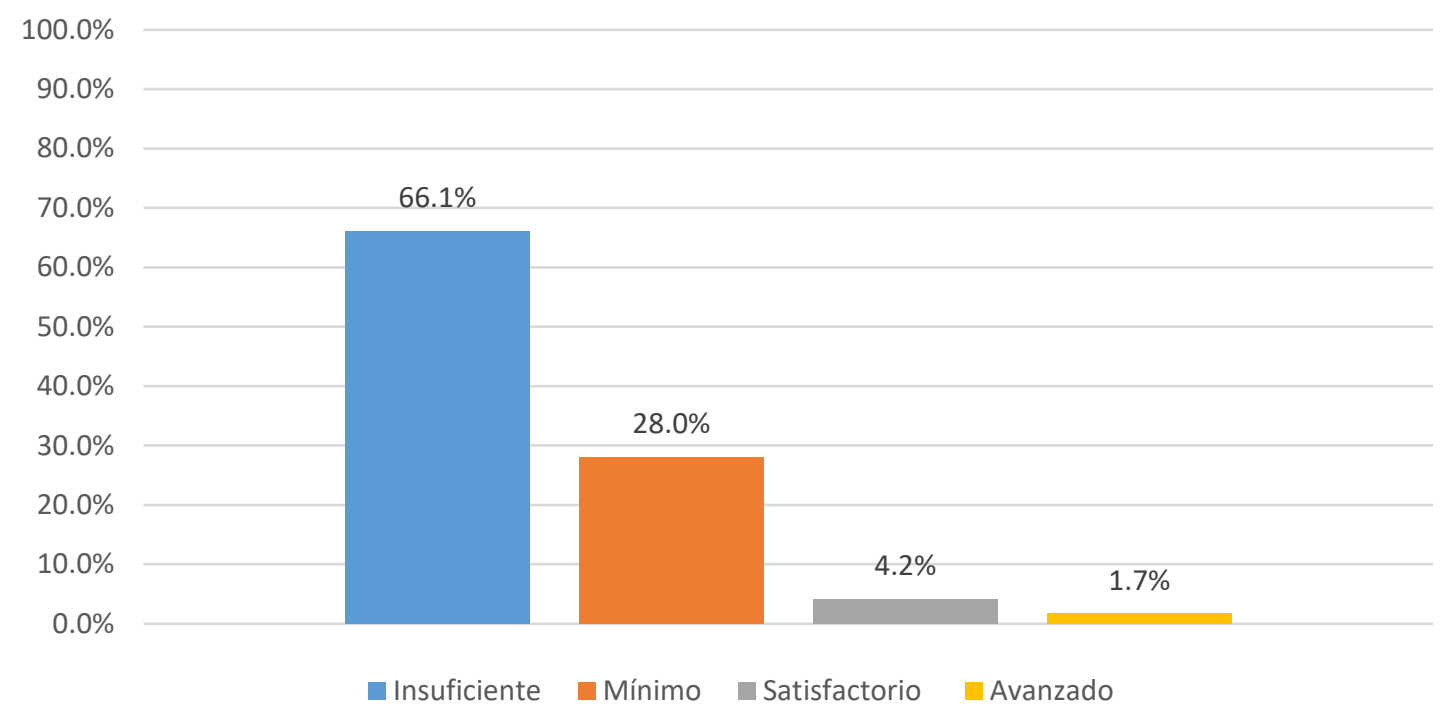

Figura 19

Distribución estudiantil por niveles de desempeño de los estudiantes de grado $10^{\circ}$ de EMV, de acuerdo a la competencia científica Indagación

Grado: $10^{\circ}$

$100.0 \%$

$90.0 \%$

$80.0 \%$

$70.0 \%$

$60.0 \%$

$50.0 \%$

$40.0 \%$

$30.0 \%$

$20.0 \%$

$10.0 \%$

$0.0 \%$

$68.2 \%$

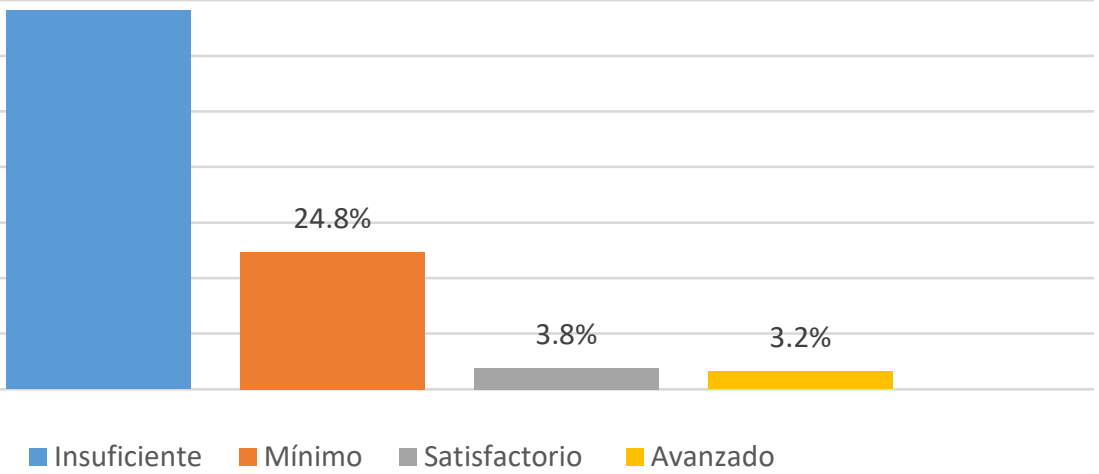




\section{Figura 20 \\ Distribución estudiantil por niveles de desempeño de los estudiantes de grado $11^{\circ}$ de $E M V$, de acuerdo con la competencia científica Indagación}

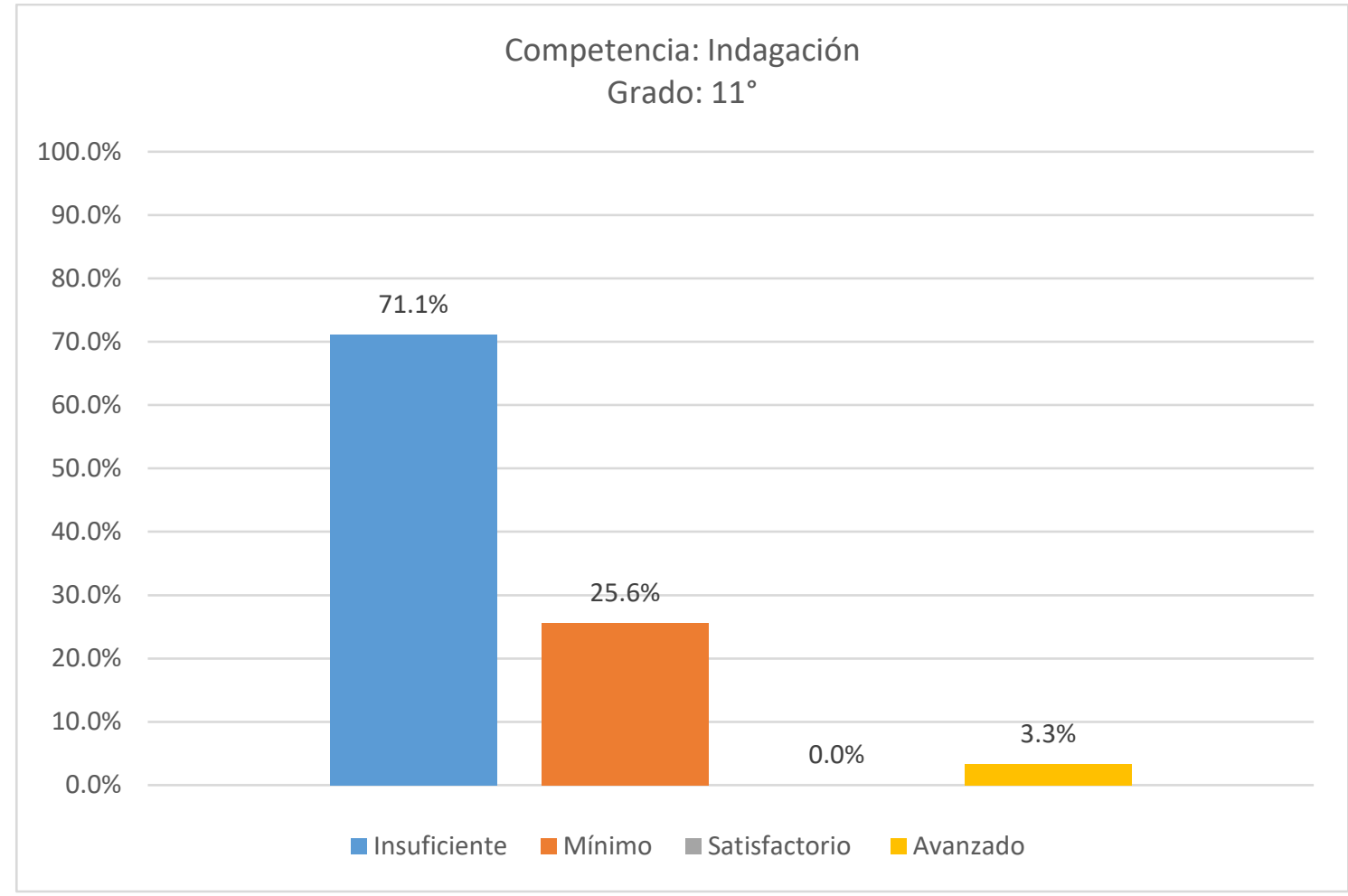

Nota. De acuerdo a la información de las gráficas presentadas, en promedio el 93,7\% de los estudiantes de todos los grados se ubicó en los niveles de desempeño inferior (insuficiente y mínimo), que la mayoría de los estudiantes de estos grados no posee la habilidad requerida para desarrollar satisfactoriamente el proceso de indagación en ciencias para el grado educativo en el cual se encuentra.

En la figura 21 se muestra el comportamiento de la población estudiantil en la competencia científica indagación, que presenta los niveles de menor desempeño (barras de color azul y naranja) y la disminución de la población ubicada en los niveles de desempeño superior (barras de color gris y amarillo) en referencia con la competencia para la explicación de indagación, en mayor medida que los resultados identificados para la competencia de uso comprensivo del conocimiento científico y de forma similar a la competencia de explicación de fenómenos. 


\section{Figura 21}

Síntesis de la distribución porcentual de la población estudiantil en relación a la competencia cientifica indagación

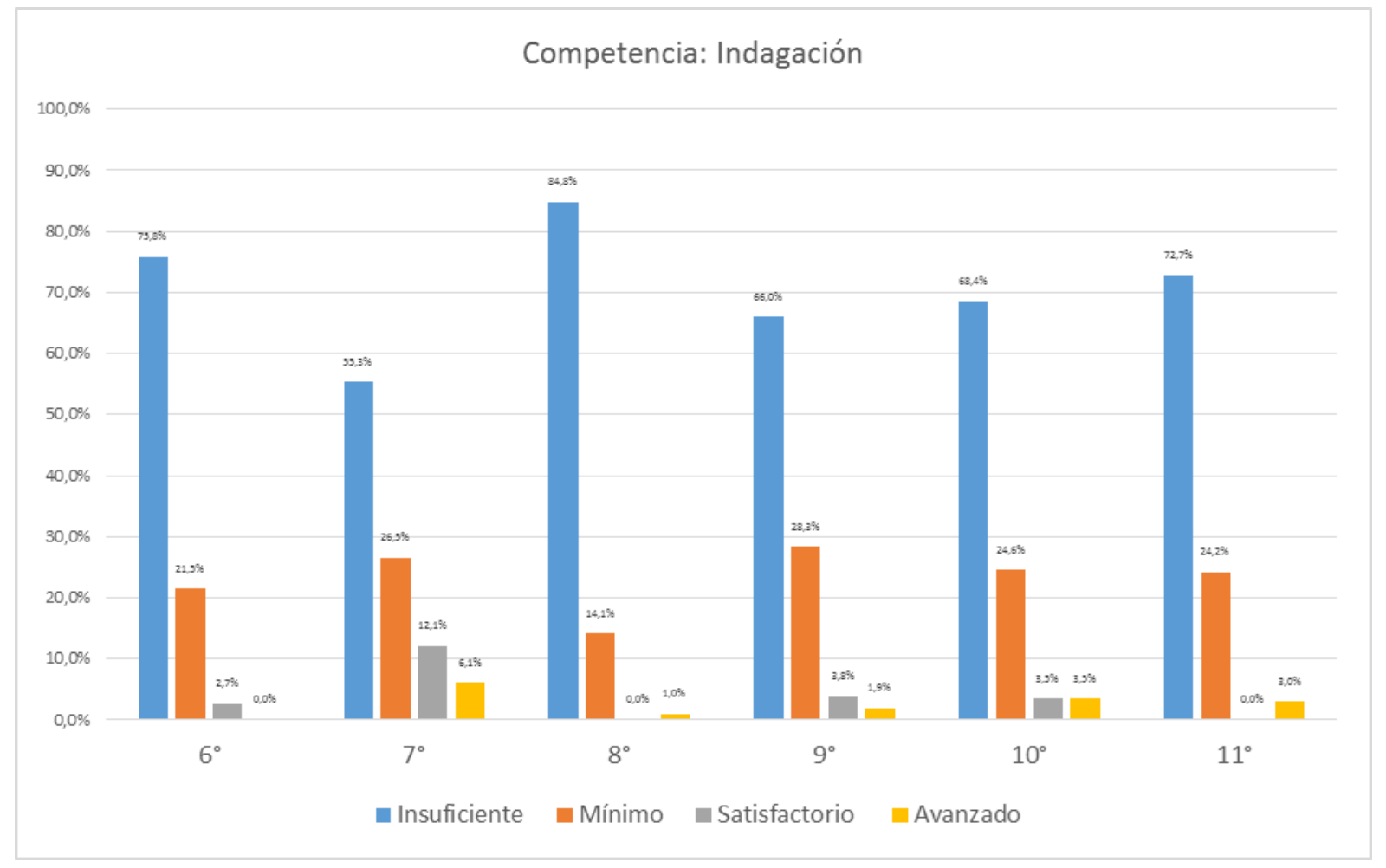

\section{DISCUSIONES}

Teniendo en cuenta los resultados obtenidos, se puede afirmar que las características de los desempeños académicos de los estudiantes discriminados por las competencias científicas descritas reflejan la consecuencia de la naturaleza de los procesos de planificación, práctica de aula y evaluación institucional desarrollados. Estos evidenciaron los resultados producto de la enseñanza basada prioritariamente en conocimientos declarativos y conceptuales a través de la estimulación de la memoria y ejercicios de tratamiento teórico y textual en detrimento de actividades y ejercicios de análisis, síntesis, reflexión, explicación e indagación por las causalidades, naturalezas y consecuencias de los fenómenos naturales.

Está marcada tendencia se evidencia de manera progresiva durante el tránsito de los grados de la básica secundaria hasta la media, principalmente en el desempeño estudiantil de las competencias explicación de fenómenos e indagación, aun cuando el resultado de la evaluación de los aprendizajes no es del todo satisfactorio en relación a la competencia de uso comprensivo del conocimiento científico.

Se puede concluir, de acuerdo con Davini (2015) y Litwin (2008) que el enfoque educativo predominante en la práctica pedagógica institucional es el técnico-eficientista, a través del cual se hace diferencia entre el conocimiento teórico y práctico, donde la práctica es la aplicación de lo teórico, el cual presenta una visión de un docente técnico que se convierte en un objeto social de control, cuya función es únicamente la de enseñar. 
El trabajo didáctico y pedagógico aislado de los avances e iniciativas epistemológicas históricas de las ciencias, de los diferentes paradigmas psicoeducativos, la evolución de los aspectos personales y sociales de las ciencias plasmadas en los diversos paradigmas que han marcado este proceso (asociacionista, aprendizaje significativo, epistemología genética de Piaget, movimiento de concepciones alternativas, el paradigma constructivista de la educación, entre otros).

Este supone la continuidad de premisas curriculares ingenuas, producto de la ausencia o deficiente reflexión didáctica y pedagógica de sus autores, que asumen estos procesos más por el peso de la tradición. Al respecto Pérez Abril y Rincón (2009) plantean que el sistema de intereses personales de los docentes, basados en las creencias y concepciones configuran las representaciones educativas de la enseñanza, el aprendizaje, los fines de la escuela y el sentido de la formación en ciencias que estos sujetos poseen y se materializan en las prácticas pedagógicas.

En este mismo marco de ideas se establece, a través de esta propuesta, la necesidad de promover un consenso académico de entre todos los integrantes de la comunidad educativa y principalmente entre docentes y directivos docentes en torno a las características de los procesos educativos desarrollados en la actualidad.

\section{CONCLUSIONES}

La experiencia de la renovación curricular en nuestro país nos muestra que un avance hacia la calidad en las prácticas pedagógicas (refiriéndose a las del lenguaje, pero perfectamente aplicables a las ciencias naturales 0 cualquier otra disciplina), y de las prácticas educativas en general.

No se garantiza con contar con unos programas curriculares bien diseñados, si la práctica pedagógica de los colectivos de docentes no está atravesada por el estudio y reflexión permanentes sobre los enfoques, procesos y competencias fundamentales que determinan el desarrollo integral de los estudiantes.

De acuerdo a esta premisa difícilmente se avanzará hacia procesos de calidad, es decir, sin un ejercicio didáctico fundamentado teórica y reflexivamente mediante la continua construcción, evolución y progresión académica, producto de la investigación educativa. Además de su perspectiva histórica, la evolución de sus paradigmas, el peso de la tradición, sus crisis y las concepciones alternativas.

En este sentido las principales conclusiones producto de los hallazgos producto del trabajo didáctico descriptivo, comprensivo y explicativo de la práctica pedagógica del área de química, esbozados en el presente trabajo, no difieren de otros estudios investigativos.

Como consecuencia de la dinámica y naturaleza de la práctica pedagógica institucional se estableció el deficiente nivel de desarrollo y adquisición por parte de los estudiantes de educación básica secundaria y media en función de las tres competencias científicas evaluadas, teniendo en cuenta que la mayor distribución poblacional se enmarcó en los niveles de desempeño insuficiente y mínimo. Y mucho más preocupante lo representa el hecho de que a medida que transcurre el transido desde los grados inferiores a los superiores estos desempeños se agudizan, aumentando proporcionalmente la población en el menor nivel de desempeño. 
Se puede concluir que el enfoque educativo predominante en la práctica pedagógica institucional es el técnicoeficientista a través del cual se hace diferencia entre el conocimiento teórico y práctico, donde la práctica es la aplicación de lo teórico.

Se presenta una visión de un docente técnico que se convierte en un objeto social de control, cuya función es únicamente la de enseñar, y los resultados actuales del desempeño y desarrollo de competencias científicas, producto del desarrollo de este tipo de prácticas.

Se denotan deficientes aprendizajes y evidencias prácticas de desempeño de los estudiantes y limitados aprendizajes en la medida que transitan de un grado académico a otro superior, limitando el empoderamiento personal y colectivo para hacer frente al contexto mundial y poder participar activamente en su comprensión, trasformación y mejoramiento.

\section{Conflicto de intereses / Competing interests:}

$\mathrm{El}$ autor declara que no incurre en conflictos de intereses.

\section{Rol de los autores / Authors Roles:}

No aplica

\section{Fuentes de financiamiento / Funding:}

El autor declara que no recibió un fondo específico para esta investigación.

\section{Aspectos éticos / legales; Ethics / legals:}

El autor declara no haber incurrido en aspectos antiéticos, ni haber omitido aspectos legales en la realización de la investigación.

\section{REFERENCIAS}

Arbeláez Sánchez, L. C., Díaz Barragán, N. A., Sierra Olarte, A. S., Riveros Gaona, O. L., \& Bayona, A. C. (2013). Secuencias Didácticas en Ciencias Naturales, Educación Básica Primaria, Ciencias - Primaria. Programa fortalecimiento de la cobertura con calidad para el sector educativo rural PER II. Ministerio de Educación NAcional de Colombia MEN. https://www.mineducacion.gov.co/1759/articles329722_archivo_pdf_ciencias_primaria.pdf

Banco Mundial. (2008). La calidad de la educación en Colombia: un análisis y programa de política. Enfoque, 1, 1-8. https://www.icfes.gov.co/documents/20143/237052/Calidad educacion Colombia un analisis y opciones programa de politica enfoque Banco Mundial.pdf

Blanchar Añez, F. J. (2020). Características de la práctica pedagógica en el área de Química. Revista Científica, 37(1), 30-57. https://revistas.udistrital.edu.co/index.php/revcie/article/view/14855/15427

Congreso de la República de Colombia. (1994). Ley 115 de Febrero 8 de 1994. Por la cual se expide la ley general de educación. https://www.mineducacion.gov.co/1621/articles-85906_archivo_pdf.pdf 
Davini, M. (2015). La formación en la práctica docente. Educación, Formación e Investigación., 1(2).

Delors, J., Al Mufti, I., Amagi, I., Carneiro, R., Chung, F., Geremek, B., Gorham, W., Kornhauser, A., Manley, M., Padrón Quero, M., Savané, M.-A., Singh, K., Stavenhagen, R., Won Suhr, M., \& Nanzhao, Z. (1996). La educación encierra un tesoro. Informe a la UNESCO de la Comisión Internacional para la educación del siglo XXI. In E. UNESCO (Ed.), La educación encierra un tesoro. https://unesdoc.unesco.org/ark:/48223/pf0000109590_spa

Hernandes, J. T., Figueroa, M., Carula, C., Patiño, M. I., Tafur, M., \& Duque, M. (2004). Pequeños científicos, una aproximación sistémica al aprendizaje de las ciencias en la escuela. Revista de Estudios Sociales, 19, 51-36. https://www.redalyc.org/pdf/815/81501904.pdf

Hernández Sampieri, R., Fernandez Collado, C., \& Baptista Lucio, P. (2010). Metodología de la Investigación (McGraw-Hill/ Interamericana Editores S.A. DE C.V. (ed.)).

Instituco Colombiano para la Evaluación de la Calidad de la Educación ICFES. (2016). Informe nacional de resultados PISA 2012. https://www.icfes.gov.co/documents/20143/237187/Informe nacional de resultados pisa 2012.pdf

Litwin, E. (2008). El oficio de enseñar. Condiciones y contextos (Editorial). https://amsafe.org.ar/wpcontent/uploads/Litwin-El_Oficio_de_Ensenar.pdf

Ministerio de Educación Nacional de Colombia MEN. (1994). Decreto 1743 de 1994. por el cual se instituye el Proyecto de Educación Ambiental para todos los niveles de educación formal, se fijan criterios para la promoción de la educación ambiental no formal e informal y se establecen los mecanismos de coordinació. https://www.funcionpublica.gov.co/eva/gestornormativo/norma_pdf.php?i=1301

Ministerio de Educación Nacional de Colombia MEN. (1998). Lineamientos curriculares de ciencas naturales y educación ambiental. https://uww.mineducacion.gov.co/1621/articles-89869_archivo_pdf5.pdf

Ministerio de Educación Nacional de Colombia MEN. (2006). Estándares Básicos de Competencias en Lenguaje, Matemáticas, Ciencias y Ciudadanas. Guía sobre lo que los estudiantes deben saber y saber hacer con lo que aprenden. Editorial MEN. https://www.mineducacion.gov.co/1621/articles-340021_recurso_1.pdf

Ministerio de Educación Nacional de Colombia MEN. (2008). Seria de Guias 34. Guia para el mejoramiento institucional. De la autoevaluación al plan de mejoramiento. MEN. https://www.mineducacion.gov.co/1621/articles-177745_archivo_pdf.pdf

Ministerio de Educación Nacional de Colombia MEN. (2014). Documento Guía: Docente de básica secundaria y media en ciencias naturales y educación ambiental (Química). MEN. https://www.mineducacion.gov.co/1759/articles-342767_recurso_17.pdf

Ministerio de Educación Nacional de Colombia MEN. (2016a). Derechos Básicos de Aprendizaje de Ciencias 
Naturales. https://eduteka.icesi.edu.co/pdfdir/DBA_CNaturales.pdf

Ministerio de Educación Nacional de Colombia MEN. (2016b). Matriz de Referencia de Ciencias Naturales. https://www.saberintensamente.com/_files/ugd/758b3d_8dcac753725c4f6ca2feacb584486c5f.pdf

Ministerio de Educación Nacional de Colombia MEN. (2017). Caminos de lectura y escritura. Secuencias didácticas para los grados transición y $11^{\circ}$. https://colombiaaprende.edu.co/sites/default/files/files_public/plan-lectura-2021/manuales-ycartillas/Caminos_de_lectura_y_escritura_secuencias_didacticas_para_los_grados_transición_y_1.pdf Organización de las Naciones Unidades para la Educación, la C. y la C. U. (2013). Tercer Estudio Regional Comparativo y Explicativo TERCE. Analisis curricular. https://www.anep.edu.uy/sites/default/files/images/Archivos/publicacionesdirecciones/DSPE/lece/terce/Analisis Curricular_TERCE_2013.pdf

Pérez Abril, M., \& Rincón, G. (2009). Actividad, Secuencia Didáctica y Pedagogía por Proyectos: Tres Alternativas para la Organización del trabajo Didáctico en el Campo del lenguaje. Organización de las Naciones Unidas para la Educación, la Ciencia y la Cultura UNESCO y el Centro Regional para el Fomento del Llibro en América Latina CERLALC.

Tobón, S., Pimienta Prieto, J. H., Antonio, J., \& García Fraile, J. A. (2010). Aprendizaje y Evaluación de Competencias. In Pearson Educación de México, S.A. de C.V. (Pearson Ed). http://files.ctezona141.webnode.mx/200000004-8ed038fca3/secuencias-didacticastobon120521222400-phpapp02.pdf 